\title{
Estimation of the firing behaviour of a complete motoneuron pool by combining EMG signal decomposition and realistic motoneuron modelling
}

Abbreviated title: Estimation of the firing behaviour of a complete motoneuron pool

\author{
Arnault Caillet ${ }^{1}$, Andrew T.M. Phillips ${ }^{1}$, Dario Farina ${ }^{2, *}$, Luca Modenese ${ }^{1, *}$ \\ ${ }^{1}$ Department of Civil and Environmental Engineering, Imperial College London, SW7 2AZ, UK \\ ${ }^{2}$ Department of Bioengineering, Imperial College London, SW7 2AZ, UK \\ Email: arnault.caillet17@imperial.ac.uk \\ * These authors have equal contributions and share the senior authorship
}

NUMBER OF PAGES: 54

NUMBER OF FIGURES: 16

NUMBER OF TABLES: 3

CONFLICT OF INTEREST STATEMENT: The authors declare no competing financial interests.

ACKNOWLEDGEMENTS: AC was supported by the Skempton Scholarship and LM by an Imperial College Research Fellowship granted by Imperial College London. 


\section{Abstract}

Our understanding of the firing behaviour of motoneuron (MN) pools during human voluntary muscle contractions is currently limited to electrophysiological findings from animal experiments extrapolated to humans, mathematical models of $\mathrm{MN}$ pools not validated for human data, and experimental results obtained from EMG decomposition. These approaches are limited in accuracy or provide information on only small partitions of the MN population. Here, we propose a method based on the combination of high-density EMG (HDEMG) data and realistic modelling for predicting the behaviour of entire pools of motoneurons in humans. The method builds on a physiologically realistic model of a MN pool which predicts, from the experimental spike trains of a smaller number of individual MNs identified from decomposed HDEMG signals, the unknown recruitment and firing activity of the remaining unidentified MNs in the complete MN pool. The MN pool model is described as a cohort of leaky fireand-integrate (LIF) models of MNs scaled by a physiologically realistic distribution of MN electrophysiological properties and driven by a spinal synaptic input, both derived from decomposed HDEMG data. The MN spike trains and effective neural drive to muscle, predicted with this method, have been successfully validated experimentally. Representative applications of the method are also presented for the prediction of activity-dependant changes in $\mathrm{MN}$ intrinsic properties and in $\mathrm{MN}$ driven neuromuscular modelling. The proposed approach provides a validated tool for neuroscientists, experimentalists, and modelers to infer the firing activity of $\mathrm{MNs}$ that cannot be observed experimentally, investigate the neurophysiology of human $\mathrm{MN}$ pools, support future experimental investigations, and advance neuromuscular modelling for investigating the neural strategies controlling human voluntary contractions.

\section{Author Summary}

Our experimental understanding of the firing behaviour of motoneuron (MN) pools during human voluntary muscle contractions is currently limited to the observation of small samples of active MNs obtained from EMG decomposition. EMG decomposition therefore provides an important but incomplete description of the role of individual MNs in the firing activity of the complete MN pool, which limits our understanding of the neural strategies of the whole $\mathrm{MN}$ pool and of how the firing activity of each MN contributes to the neural drive to muscle. Here, we combine decomposed highdensity EMG (HDEMG) data and a physiologically realistic model of $M N$ population to predict the unknown recruitment and firing activity of the remaining unidentified MNs in the complete MN pool. 
bioRxiv preprint doi: https://doi.org/10.1101/2022.02.21.481337; this version posted February 22, 2022. The copyright holder for this preprint (which was not certified by peer review) is the author/funder, who has granted bioRxiv a license to display the preprint in perpetuity. It is made available under aCC-BY 4.0 International license.

33 In brief, an experimental estimation of the synaptic current is input to a cohort of MN models, which 34 are calibrated using the available decomposed HDEMG data, and predict the MN spike trains fired by 35 the entire MN population. This novel approach is experimentally validated and applied to muscle force prediction from neuromuscular modelling, and to investigate neurophysiological properties of the human MN population during voluntary contractions. 


\section{INTRODUCTION}

During voluntary muscle contractions, pools of spinal alpha-motoneurons (MNs) convert the synaptic input they receive into a neural command that drives the contractile activity of the innervated muscle fibres, determining limb motion. Identifying the recruitment and firing dynamics of MNs is fundamental for understanding the neural strategies controlling human voluntary motion, with applications in sport sciences (Felici \& Del Vecchio, 2020; Watanabe, K. et al., 2021; Maeda et al., 2021), and neurological and musculoskeletal rehabilitation (Jordanić et al., 2016; Fang et al., 2020; Pilkar et al., 2020; Kisiel-Sajewicz et al., 2020; Nishikawa et al., 2021). Determining the MN-specific contributions to the MN population activity also allows more realistic control of neuromuscular models (Callahan et al., 2013; Potvin \& Fuglevand, 2017; Kim \& Kim, 2018; Carriou et al., 2019), investigation of muscle neuromechanics (Waasdorp et al., 2021; Martinez-Valdes et al., 2021), prediction of limb motion from MN-specific behaviour (Chen et al., 2020), or improvement in human-machine interfacing and neuroprosthetics (Farina et al., 2017; Farina et al., 2021).

Our understanding of $\mathrm{MN}$ pool firing behaviour during human voluntary tasks is however currently limited. While the $\mathrm{MN}$ membrane afterhyperpolarization and axonal conduction velocity can be inferred from indirect specialized techniques (Freund et al., 1975; Dengler et al., 1988), most of the other electro-chemical MN membrane properties and mechanisms that define the $\mathrm{MN}$ recruitment and discharge behaviour cannot be directly observed in humans in vivo. Analysis of commonly adopted bipolar surface EMG recordings, which often lump the motor unit (MU) trains of action potentials into a single signal assimilated as the neural drive to muscle, cannot advance our understanding of the MN pool activity at the level of single MNs. Our experimental knowledge on the remaining MN membrane properties in mammals is therefore obtained from in vitro and in situ experiments on animals (Heckman \& Enoka, 2012). The scalability of these mechanisms to humans is debated (Manuel et al., 2019) due to a systematic inter-species variance in the MN electrophysiological properties in mammals (Highlander et al., 2020). Decomposition of high-density EMG (HDEMG) or intramuscular EMG (iEMG) signals (Holobar \& Farina, 2014; Negro, Muceli et al., 2016) allows the in vivo decoding in human muscles of the firing activity of individual active motoneurons during voluntary contractions and provide a direct window on the internal dynamics of MN pools. Specifically, the non-invasive EMG approach to $\mathrm{MN}$ decoding has recently advanced our physiological understanding of the neurophysiology of human MU pools and of the interplay between the central nervous system and the muscle contractile machinery (Del Vecchio et al., 2019; Oliveira \& Negro, 2021) 
bioRxiv preprint doi: https://doi.org/10.1101/2022.02.21.481337; this version posted February 22, 2022. The copyright holder for this preprint (which was not certified by peer review) is the author/funder, who has granted bioRxiv a license to display the preprint in perpetuity. It is made available under aCC-BY 4.0 International license.

Yet, the activity of all the MNs constituting the complete innervating MN population of a muscle cannot be identified with this technique. High-yield decomposition typically detects at most 30-40 MNs (Del Vecchio et al., 2020), while MU pools typically contain hundreds of MUs in muscles of the hindlimb (Heckman \& Enoka, 2012). The small sample of recorded MNs is besides not representative of the continuous distribution of the MN electrophysiological properties in the complete $\mathrm{MN}$ pool with a bias towards the identification of mainly high-threshold MUs. The samples of spike trains obtained from signal decomposition therefore provide a limited description of the role of individual MNs in the firing activity of the complete $\mathrm{MN}$ pool.

To allow the investigation and description of specific neurophysiological mechanisms of the complete MN population, some studies have developed mathematical frameworks and computational models of pools of individual MNs. These MN pool models have provided relevant insights for interpreting experimental data (Fuglevand et al., 1993; Dideriksen et al., 2010), investigating the MN pool properties and neuromechanics (Cisi \& Kohn, 2008; Negro \& Farina, 2011; Farina et al., 2014), neuromuscular mechanisms (Watanabe, R. N. et al., 2013; Potvin \& Fuglevand, 2017), and the interplay between muscle machinery and spinal inputs (Dideriksen et al., 2011). However, none of these MN pool models have been tested with experimental input data, instead either receiving artificial gaussian noise (Negro \& Farina, 2011), sinusoidal (Farina et al., 2014) or ramp (Fuglevand et al., 1993) inputs, inputs from interneurons (Cisi \& Kohn, 2008) or feedback systems (Dideriksen et al., 2010). These MN pool models have therefore never been tested in real conditions of voluntary muscle contraction. The forward predictions of MN spike trains or neural drive to muscle obtained in these studies were consequently not or indirectly validated against experimental recordings.

The MN-specific recruitment and firing dynamics of these $\mathrm{MN}$ pool models are usually described with comprehensive or phenomenological models of MNs. The biophysical approaches (Cisi \& Kohn, 2008; Negro \& Farina, 2011; Watanabe, R. N. et al., 2013; Farina et al., 2014), which rely on a population of compartmental Hodgkin-Huxley-type MN models provide a comprehensive description of the microscopic MN-specific membrane mechanisms of the $\mathrm{MN}$ pool and can capture complex nonlinear MN dynamics (Röhrle et al., 2019). However, these models are computationally expensive and remain generic, involving numerous electrophysiological channel-related parameters for which adequate values are difficult to obtain in mammalian experiments (Bondarenko et al., 2004; Fohlmeister, 2009) and must be indirectly calibrated or extrapolated from animal measurements in human models (Smit et al., 2008). On the other hand, phenomenological models of MNs (Dideriksen et al., 2010; Raikova et al., 2018; Carriou et al., 2019) provide a simpler description of the MN pool dynamics and rely on a few parameters that can be calibrated or inferred in mammals including humans. They are inspired from the Fuglevand's formalism (Fuglevand et al., 1993), where the output MN firing frequency is the 
bioRxiv preprint doi: https://doi.org/10.1101/2022.02.21.481337; this version posted February $22,2022$. The copyright holder for this preprint (which was not certified by peer review) is the author/funder, who has granted bioRxiv a license to display the preprint in perpetuity. It is made available under aCC-BY 4.0 International license.

gaussian-randomized linear response to the synaptic drive with a $\mathrm{MN}$-specific gain. However, these phenomenological models cannot account for the $\mathrm{MN}$-specific nonlinear mechanisms that dominate the MN pool behaviour (Röhrle et al., 2019). MN leaky integrate-and-fire (LIF) models are an acceptable trade-off between Hodgkin-Huxley-type and Fuglevand-type $\mathrm{MN}$ models with intermediate computational cost and complexity, and accurate descriptions of the MN macroscopic discharge behaviour (Teeter et al., 2018). LIF models, the parameters of which are defined by MN membrane electrophysiological properties for which mathematical relationships are available (Caillet et al., 2021), moreover provide a convenient framework for a physiologically-realistic description of the MN pool. While repeatedly used for the modelling and the investigation of individual MN neural dynamics (Izhikevich, 2004; Dong et al., 2011; Negro, Yavuz et al., 2016), MN LIF models are however not commonly used for the description of MN pools.

To the authors' knowledge there is no systematic method to record the firing activity of all the MNs in a $\mathrm{MN}$ pool, or to estimate from a sample of experimental $\mathrm{MN}$ spike trains obtained from signal decomposition the firing behaviour of $\mathrm{MNs}$ that are not recorded in the $\mathrm{MN}$ pool. There is no mathematical model of a MN pool that (1) was tested with experimental neural inputs and investigated the neuromechanics of voluntary human muscle contraction, (2) involves a cohort of MN models that relies on $\mathrm{MN}$-specific profiles of inter-related $\mathrm{MN}$ electrophysiological properties, (3) is described by a physiologically-realistic distribution of $\mathrm{MN}$ properties that is species-specific and consistent with available experimental data. This limits our understanding of the neural strategies of the whole MN pool and of how the firing activity of each MN contributes to the neural drive to muscle.

In this study, a novel four-step approach is designed to predict, from the neural information of $N_{r} \mathrm{MN}$ spike trains obtained from HDEMG signal decomposition, the recruitment and firing dynamics of the $N-N_{r}$ MNs that were not identified experimentally in the investigated pool of $N$ MNs. The model of the MN pool was built upon a cohort of $N$ LIF models of MNs. The LIF parameters are derived from the available HDEMG data, are MN-specific, and account for the inter-relations existing between mammalian MN properties (Caillet et al., 2021). The distribution of $N$ MN input resistances hence obtained defines the recruitment dynamics of the $\mathrm{MN}$ pool. The $\mathrm{MN}$ pool model is driven by a common synaptic current, which is estimated from the available experimental data as the filtered cumulative summation of the $N_{r}$ identified spike trains. The MN-specific LIF models transform this synaptic current into accurate discharge patterns for the $N_{r}$ MNs experimentally identified and predict the MN firing dynamics of the $N-N_{r}$ unidentified MNs. The blind predictions of the spike trains of the $N_{r}$ identified MNs and the effective neural drive to the muscle, computed from the firing activity of the complete pool of $N$ virtual MNs, are both successfully validated against available experimental data. 
bioRxiv preprint doi: https://doi.org/10.1101/2022.02.21 481337; this version posted February $22,2022$. The copyright holder for this preprint (which was not certified by peer review) is the author/funder, who has granted bioRxiv a license to display the preprint in perpetuity. It is made available under aCC-BY 4.0 International license.

137 Neuroscientists can benefit from this proposed approach for inferring the neural activity of MNs that 138 cannot be observed experimentally and for investigating the neurophysiology of MN populations.

139 Moreover, this approach can be used by modelers to design and control realistic neuromuscular 140 models, useful for investigating the neural strategies in muscle voluntary contractions and predicting

141 muscle quantities that cannot be obtained experimentally. In this study, we provide an example for

142 this application by using the simulated discharge patterns of the complete MN pool as inputs to Hilltype models of muscle units to predict muscle force. 


\section{METHODS}

\section{Overall approach}

147 The spike trains $s p_{j}^{s i m}(t)$ elicited by the entire pool of $N$ MNs were inferred from a sample of $N_{r}$ 148 experimentally identified spike trains $s p_{i}^{\exp }(t)$ with a 4-step approach displayed in Figure 1 . The $149 N_{r}$ experimentally-identified MNs were allocated to the entire $\mathrm{MN}$ pool according to their recorded 150 force recruitment thresholds $F_{i}^{\text {th }}$ (step 1 ). The common synaptic current $I(t)$ to the MN pool was 151 estimated from the experimental spike trains $s p_{i}^{e x p}(t)$ of the $N_{r}$ MNs (step 2). A cohort of $N_{r}$ leaky152 and-fire (LIF) models of MNs, the electrophysiological parameters of which were mathematically 153 determined by the unique MN size parameter $S_{i}$, transformed the input $I(t)$ to simulate the 154 experimental spike trains $s p_{i}^{e x p}(t)$ after calibration of the $S_{i}$ parameter (step 3). The distribution of 155 the $N$ MN sizes $S(j)$ in the entire $\mathrm{MN}$ pool, which was extrapolated by regression from the $N_{r}$ 156 calibrated $S_{i}$ quantities, scaled the electrophysiological parameters of a cohort of $N$ LIF models. The $N$ 157 calibrated LIF models predicted from $I(t)$ the spike trains $s p_{j}^{s i m}(t)$ of action potentials elicited by the 158 entire pool of $N$ virtual MNs. 


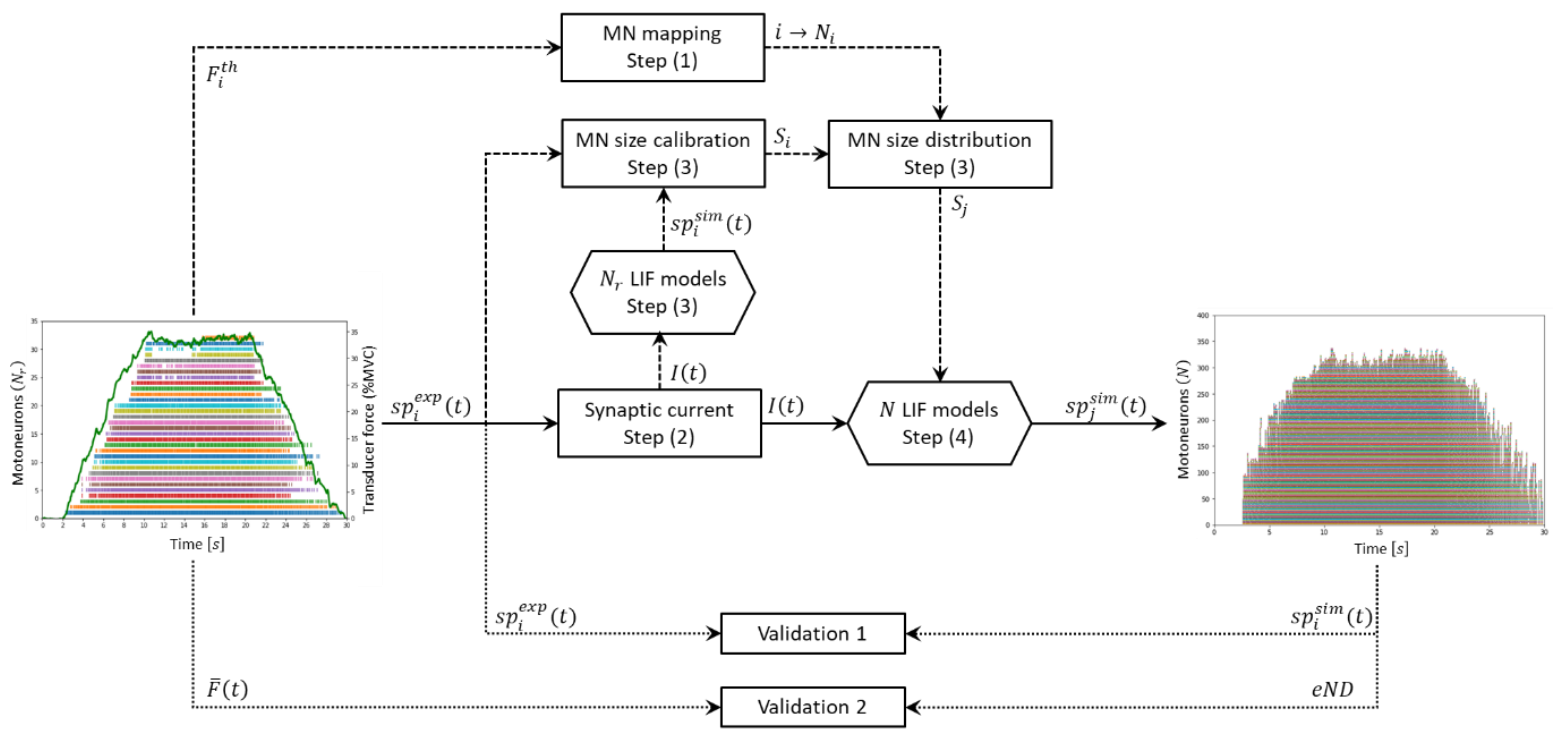

Figure 1: Four-step workflow predicting the spike trains $\operatorname{sp}_{j}^{\operatorname{sim}}(t)$ of the entire pool of $N$ MNs (right figure) from the experimental sample of $N_{r} M N$ spike trains $s p_{i}^{\text {exp }}(t)$ (left figure). Step (1): according to their experimental force thresholds $F_{i}^{\text {th }}$, each $M N$, ranked from $i=1$ to $i=N_{r}$ following increasing recruitment thresholds, was assigned the $N_{i}^{\text {th }}$ location in the complete pool of MNs ( $i \rightarrow N_{i}$ mapping). Step (2): the common synaptic current $I(t)$ to the $M N$ spool was derived from the $N_{r}$ spike trains $s p_{i}^{\text {exp }}(t)$. Step (3): using $I(t)$ as input, the size parameter $S_{i}$ of a cohort of $N_{r}$ leaky-and-fire (LIF) MN models was calibrated by minimizing the error between predicted and experimental filtered spike trains. From the calibrated $S_{i}$ and the $M N i \rightarrow N_{i}$ mapping, the distribution of $M N$ sizes $S_{j}$ in the entire pool of virtual MNs was obtained by regression. Step (4): the $S_{j}$ distribution scaled a cohort of $N$ LIF models which predicted the $M N$-specific spike trains $s p_{j}^{s i m}(t)$ of the entire pool of MNs (right). The approach was validated by comparing experimental and predicted spike trains (Validation 1) and by comparing normalized experimental force trace $\bar{F}(t)$ (Left figure, green trace) with normalized effective neural drive (Validation 2). In both figures, the MN spike trains are ordered from bottom to top in the order of increasing force recruitment thresholds.

The following assumptions were made. (1) The MU pool is idealized as a collection of $N$ independent MUs that receive a common synaptic input and possibly MU-specific independent noise. (2) In a pool of $N$ MUs, $N$ MNs innervate $N$ muscle units (mUs). (3) In our notation, the pool of $N$ MUs is ranked from $j=1$ to $j=N$, with increasing recruitment threshold. For the $N \mathrm{MNs}$ to be recruited in increasing $\mathrm{MN}$ and $\mathrm{mU}$ size and recruitment thresholds according to Henneman's size principle (Henneman, 1957; Henneman et al., 1965a; Henneman et al., 1965b; Henneman et al., 1974; Henneman, 1981; Henneman, 1985; Heckman \& Enoka, 2012; Caillet et al., 2021), the distribution of morphometric, threshold and force properties in the MN pool follows

$$
\forall j, k \in[1 ; N],(j<k) \Longrightarrow\left(S_{j}<S_{k} \Leftrightarrow I_{j}^{t h}<I_{k}^{t h} \Leftrightarrow I R_{j}<I R_{k} \Leftrightarrow F_{j}^{t h}<F_{k}^{t h} \Leftrightarrow f_{i s o, j}^{\max }<f_{i s o, k}^{\max }\right)
$$

181 where $S$ is the $\mathrm{MN}$ surface area, $I^{\text {th }}$ the MN current threshold for recruitment, $I R$ the MU innervation ratio defining the $\mathrm{MU}$ size, $F^{t h}$ is the $\mathrm{MU}$ force recruitment threshold, and $f_{\text {iso }}^{\max }$ is the $\mathrm{MU}$ maximum 
isometric force. (4) The MN-specific electrophysiological properties are mathematically defined by the MN size $S$ (a et al. 2021). This extends the Henneman's size principle to:

$$
\forall j, k \in[1 ; N],(j<k) \Rightarrow\left(S_{j}<S_{k} \Leftrightarrow C_{j}<C_{k} \Leftrightarrow R_{j}>R_{k} \Leftrightarrow \tau_{j}>\tau_{k}\right)
$$

Where $C$ is the $\mathrm{MN}$ membrane capacitance, $R$ the $\mathrm{MN}$ input resistance and $\tau$ the $\mathrm{MN}$ membrane time constant.

\section{Experimental data}

The four sets of experimental data used in this study, named as reported in the first column of Table 1 , provide the time-histories of recorded $\mathrm{MN}$ spike trains $\operatorname{sp}_{i}^{\exp }(t)$ and whole muscle force trace $F(t)$ (left panel in Figure 1), and were obtained from the studies (Hug, Avrillon et al., 2021; Hug, Del Vecchio et al., 2021) and (Del Vecchio et al., 2019; Del Vecchio et al., 2020), as open-source supplementary material and personal communication, respectively. In these studies, the HDEMG signals were recorded with a sampling rate $f_{s}=2048 \mathrm{~Hz}$ from the Tibialis Anterior (TA) and Gastrocnemius Medialis (GM) human muscles during trapezoidal isometric contractions. As displayed in Figure 2, the trapezoidal force trajectories are described in this study by the $t_{t r_{0 \rightarrow 5}}$ times reported in Table 1 , as a zero force in $\left[t_{t r 0} ; t_{t r 1}\right]$, a ramp of linearly increasing force in $\left[t_{t r 1} ; t_{t r 2}\right]$, a plateau of constant force in $\left[t_{t r 2} ; t_{t r 3}\right]$, a ramp of linearly decreasing force in $\left[t_{t r 3} ; t_{t r 4}\right]$, and a zero force in $\left[t_{t r 4} ; t_{t r 5}\right]$.

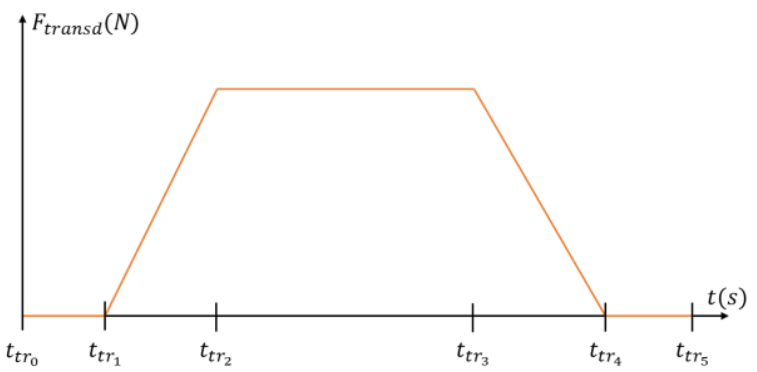

Figure 2: Definition of times $t_{t_{i}}$ that describe the trapezoidal shape of the muscle isometric contraction.

Table 1:The four experimental datasets processed in this study. $N_{r}$ spike trains are identified per dataset during trapezoidal contractions of the Tibialis Anterior (TA) or Gastrocnemius Medialis (GM) muscles. The trapezoidal force trace is described by times $t_{t r_{i}}$ in seconds up to a dataset-specific level of maximum voluntary contraction (\%MVC).

\begin{tabular}{|c|c|c|c|c|c|c|c|c|c|c|}
\hline Dataset & Muscle & $\% \mathrm{MVC}$ & $t_{t r 0}$ & $t_{t r 1}$ & $t_{t r 2}$ & $t_{t r 3}$ & $t_{t r 4}$ & $t_{t r 5}$ & $N_{r}$ & Reference paper \\
\hline$D T A_{35}$ & TA & 35 & 0 & 2.2 & 10.6 & 20.5 & 30 & 30 & 32 & $\begin{array}{c}\text { (Del Vecchio et al., 2019; Del } \\
\text { Vecchio et al., 2020) }\end{array}$ \\
\hline$H T A_{35}$ & TA & 35 & 0 & 2.1 & 10.5 & 20.5 & 30 & 33 & 21 & \multirow{3}{*}{$\begin{array}{l}\text { (Hug, Avrillon et al., 2021; } \\
\text { Hug, Del Vecchio et al., 2021) }\end{array}$} \\
\hline$H T A_{50}$ & TA & 50 & 0 & 1.6 & 12 & 21.8 & 34.5 & 35 & 14 & \\
\hline$H G M_{30}$ & GM & 30 & 0 & 3.1 & 9.1 & 28 & 33.5 & 107 & 27 & \\
\hline
\end{tabular}


The HDEMG signals were decomposed with blind-source separation techniques and $N_{r} \mathrm{MN}$ spike trains $s p_{i}^{e x p}(t)$ were identified. In this study, the experimental $N_{r}$ MNs were ranked from $i=1$ to $i=N_{r}$ in the order of increasing recorded force recruitment thresholds $F_{i}^{t h}$, i.e. $\forall i \in\left[1 ; N_{r}\right], F_{i}^{t h}<F_{i+1}^{t h}$. The sample time of the $\mathrm{k}^{\text {th }}$ firing event of the $\mathrm{i}^{\text {th }}$ identified $\mathrm{MN}$ is noted as $f t_{i}^{k}$, and the binary spike train of the $i^{\text {th }}$ identified MN was mathematically defined as:

$$
\left\{\begin{array}{l}
s p_{i}^{\exp }\left(t=f t_{i}^{k}\right)=1 \\
s p_{i}^{\exp }\left(t \neq f t_{i}^{k}\right)=0
\end{array}\right.
$$

The train of instantaneous discharge frequency $I D F_{i}(t)$ of the $\mathrm{i}^{\text {th }}$ identified $\mathrm{MN}$ was computed between firing times $f t_{i}^{k}$ and $f t_{i}^{k+1}$ as:

$$
\left\{\begin{array}{c}
I D F_{i}\left(t=f t_{i}^{k}\right)=\frac{1}{f t_{i}^{k+1}-f t_{i}^{k}} \\
I D F_{i}\left(t \neq f t_{i}\right)=0
\end{array}\right.
$$

The IDFs were moving-average filtered by convolution with a Hanning window of length $400 \mathrm{~ms}$ (De Luca, C. J. et al., 1982), yielding the continuous filtered instantaneous discharge frequencies (FIDFs) for all $N_{r}$ identified MNs.

\section{Approximation of the TA and GM MU pool size}

The typical number $N$ of MUs was estimated for the TA muscle from cadaveric studies (Feinstein et al., 1955), statistical methods (Trojaborg et al., 2002), decomposed-enhanced spike-triggered-averaging (DESTA) approaches (Van Cutsem, M. et al., 1997; McNeil et al., 2005; Boe et al., 2009; Power et al., 2010; Hourigan et al., 2015; Piasecki et al., 2016), and adapted multiple point stimulation methods (Xiong et al., 2008) in 20-80-year-old human subjects. Because of method-specific limitations (Gooch et al., 2014), results across methods varied substantially, with estimates for $N$ of, respectively, 445, 194, 190, 188 and 300 MUs for the TA muscle. DESTA methods systematically underestimate the innervation ratio due to the limited muscle volume covered by the surface electrodes. Cadaveric approaches rely on samples of small size and arbitrarily distinguish alpha from gamma MNs. Twitch torque measurements are an indirect method for estimating $N$. Accounting for these limitations, we estimated $N_{T A}=400 \mathrm{MUs}$ in a typical adult TA muscle. Assuming 200,000 fibres in the TA muscle (Henriksson-Larsén et al., 1983; Henriksson-Larsén, 1985), $N_{T A}=400$ yields a mean of 500 fibres per TA MU, consistently with previous findings (Henriksson-Larsén, 1985). In two cadaveric studies (Feinstein et al., 1955; Christensen, 1959), the estimate for the GM was $N_{G M}=550$ MUs, which is 
consistent with $N_{T A}=400$ as the GM muscle volume is typically larger than TA's (Handsfield et al., 2014).

\section{Step (1): MN mapping}

In the first step of the approach overviewed in Figure 1, the $N_{r}$ experimentally identified MNs were allocated to the entire pool of $N$ MNs according to their recorded force recruitment thresholds $F_{i}^{t h}$. Three studies measured in the human TA muscle in vivo the force recruitment thresholds $F^{\text {th }}$ of MUs, given as a percentage of the maximum voluntary contraction (\%MVC) force, for 528 (Van Cutsem, M. et al., 1997), 256 (Van Cutsem, Michaël et al., 1998), and 302 (Feiereisen et al., 1997) MUs. Other studies investigated TA MU pools but reported small population sizes (Desmedt \& Godaux, 1977) and/or did not report the recruitment thresholds (Andreassen \& Arendt-Nielsen, 1987; Vander Linden et al., 1991; Connelly et al., 1999).

We digitized the scatter plot in Figure 3 in Van Cutsem et al. (1997) using the online tool WebPlotDigitizer (Ankit, 2020). The normalized MU population was partitioned into 10\%-ranges of the values of $F^{\text {th }}$ (in \%MVC), as reported in Van Cutsem et al. (1998) and Feiereisen et al. (1997). The distributions obtained from these three studies were averaged. The normalized frequency distribution by $10 \% \mathrm{MVC}$-ranges of the $F^{\text {th }}$ quantities hence obtained was mapped to a pool of $N$ MUs, providing a step function relating each $j^{\text {th }} \mathrm{MU}$ in the $\mathrm{MU}$ population to its $10 \%$-range in $F^{\text {th }}$. This step function was least-squares fitted by a linear-exponential trendline $F^{t h}(j)=k_{1} \cdot\left(k_{2} \cdot \frac{j}{N}+\Delta_{\mathrm{F}}^{\left(\frac{j}{N}\right)^{k_{3}}}\right), j \in \llbracket 1 ; N \rrbracket$, providing a continuous frequency distribution of TA MU recruitment thresholds in a MU pool that reproduces the available literature data. Simpler trendlines, such as $F^{\text {th }}(j)=k_{1} \cdot \Delta_{\mathrm{F}}^{\left(\frac{j}{N}\right)^{k_{3}}}$ (Fuglevand et al., 1993), returned fits of lower $r^{2}$ values. According to the three studies and to Heckman \& Enoka (2012), a $\Delta_{\mathrm{F}}=120$-fold range in $F^{\text {th }}$ was set for the TA muscle, yielding $F^{t h}(N)=90 \% \mathrm{MVC}=\Delta_{\mathrm{F}}$. $F^{t h}(1)$, with $F^{t h}(1)=0.75 \% \mathrm{MVC}$. Finally, the equation $F^{t h}\left(N_{i}\right)=k_{1} \cdot\left(k_{2} \cdot \frac{N_{i}}{N}+\Delta_{\mathrm{F}}^{\left(\frac{N_{i}}{N}\right)^{k_{3}}}\right)=F_{i}^{t h}$ was solved for the variable $N_{i}$ for all $N_{r}$ identified MUs for which the experimental threshold $F_{i}^{\text {th }}$ was recorded. The $N_{r}$ identified MUs were thus assigned the $N_{i}^{t h}$ locations of the complete pool of $N$ MUs ranked in order of increasing $F^{\text {th }}$ :

$$
i \in \llbracket 1 ; N_{r} \rrbracket \rightarrow N_{i} \in \llbracket 1 ; N \rrbracket
$$


When considering a 100-ms electromechanical delay between $\mathrm{MN}$ recruitment time and onset of muscle unit force, the mapping $i \in \llbracket 1 ; N_{r} \rrbracket \rightarrow N_{i} \in \llbracket 1 ; N \rrbracket$ did not substantially change. It was therefore simplified that a $\mathrm{MN}$ and its innervated muscle unit were recruited at the same time, and the $i \in \llbracket 1 ; N_{r} \rrbracket \rightarrow N_{i} \in \llbracket 1 ; N \rrbracket$ mapping derived for muscle units was extrapolated to MNs.

Considering that typically less than $30 \mathrm{MUs}$ ( $5 \%$ of the GM MU pool) can be currently identified by HDEMG decomposition in GM muscles (Del Vecchio et al., 2020), and that the few papers identifying GM MUs with intramuscular electrodes either did not report the MU $F^{\text {th }}$ (Garnett et al., 1979; Vieira et al., 2012; Kallio et al., 2013) or identified less than 24 MUs up to 100\% MVC (Ballantyne et al., 1993; Héroux et al., 2014), a GM-specific $F^{\text {th }}(j)$ distribution could not be obtained from the literature for the GM muscle. The $F^{t h}(j)$ distribution obtained for the TA muscle was therefore used for the simulations performed with the GM muscle, which is acceptable as an initial approximation based on visual comparison to the scattered data provided in these studies.

\section{Step (2): Common synaptic input current $I(t)$}

In the second step of the approach in Figure 1, the common synaptic current input to the MN pool was estimated. The cumulative spike train (CST) was obtained as the temporal binary summation of the $N_{r}$ experimental spike trains $s p_{i}^{\text {exp }}(t)$.

$$
C S T=\sum_{i} s p_{i}^{e x p}(t)
$$

The effective neural drive $(e N D)$ to the muscle was estimated by low-pass filtering the CST in the bandwidth $[0 ; 10] \mathrm{Hz}$ relevant for force generation (Negro, Yavuz et al., 2016). As the pool of MNs filters the $\mathrm{MN}$-specific independent synaptic noise received by the individual MNs and linearly transmits the common synaptic input (CSI) to the MN pool (Farina et al., 2014; Farina \& Negro, 2015), the $C S I$ was equalled to the $e N D$ in arbitrary units:

$$
\operatorname{CSI}(t)=k \cdot e N D(t)
$$

The common synaptic control (CSC) signal was obtained by low pass filtering the $C S I$ in $[0 ; 4] \mathrm{Hz}$.

This approach, which estimates the $C S I$ from the $N_{r}$ experimental spike trains is only valid if the sample of $N_{r} \mathrm{MNs}$ is 'large enough' and 'representative enough' of the complete MN pool for the linearity properties of the population of $N_{r}$ MNs to apply (Farina \& Negro, 2015). To assess if this approximation holds with the $N_{r}$ MNs obtained experimentally, the following two validations were performed. (1) The coherence $\operatorname{coher}_{\frac{N_{r}}{2}} \in[0 ; 1]$, averaged in $[1 ; 10] \mathrm{Hz}$, was calculated between two cumulative spike 
trains $C S T_{\frac{N_{r}}{2}, 1}$ and $C S T_{\frac{N_{r}}{2}, 2}$ computed from two complementary random subsets of $\frac{N_{r}}{2}$ MNs. This was repeated 20 times for random permutations of complementary subsets of $\frac{N_{r}}{2} \mathrm{MNs}$, and the $\operatorname{coher}_{\frac{N_{r}}{2}}$ values were average yielding $\operatorname{coher}_{\frac{N_{r}}{2}}$,mean $\in[0 ; 1]$. The coherence $\operatorname{coher}_{N_{r}}$ between the complete experimental sample of $N_{r}$ MNs and a virtual sample of $N_{r}$ non-identified MNs was finally estimated by reporting the pair $\left(\frac{N_{r}}{2}\right.$; $\left.\operatorname{coher}_{\frac{N_{r}}{2} \text {,mean }}\right)$ similarly to Figure 2A in Negro et al. (2016). (2) The timehistories of the normalized force $\bar{F}(t)$ and common synaptic control $\overline{C S C}$, which should superimpose if the linearity properties apply (see Figure 6 in Farina \& Negro (2015)), were compared with calculation of the normalized root-mean-square error (nRMSE) and coefficient of determination $r^{2}$. If $\operatorname{coher}_{N_{r}}>$ $0.7, r^{2}>0.7$ and $n R M S E<30 \%$, it was assumed that the sample of $N_{r}$ MNs was large and representative enough of the $\mathrm{MN}$ pool for the linearity properties to apply, and the $e N D$ was confidently assimilated as the CSI to the MN pool in arbitrary units. It must be noted that if $\operatorname{coher}_{N_{r}}<$ 1 , the linearity properties do not fully apply for the sample of $N_{r} \mathrm{MNs}$, and the CSI computed from the $N_{r}$ MNs is expected to relate to the true CSI with a coherence close but less than $\operatorname{coher}_{N_{r}}$.

303

The $C S I$ hence obtained reflects the synaptic excitatory influx, while the common synaptic current $I(t)$ is the dendritic membrane current arising from this synaptic influx. While the larger the $\mathrm{MN}$, the higher its affinity to $C S I$ (Heckman \& Enoka, 2012), it was simplified here that $C S I$ and $I(t)$ are linearly related with a constant gain $G$ across the $\mathrm{MN}$ pool. It must however be noted that the $C S I$, which was computed from a subset of the MN pool, does not capture the firing activity of the MNs that are recruited before the smallest identified $N_{1}^{t h} \mathrm{MN}$, which is recruited at time $f t_{N_{1}}^{1}$. It non-physiologically yields $\operatorname{CSI}\left(t<f t_{N_{1}}^{1}\right)=0$. Accounting for this experimental limitation, $I(t)$ was defined to remain null until the first identified $\mathrm{MN}$ starts firing at $f t_{N_{1}}^{1}$, and to non-continuously reach $I_{N_{1}}^{\text {th }}$ at $t=f t_{N_{1}}^{1}$ :

$$
I(t)=\left\{\begin{array}{c}
0 \text { if } t<f t_{N_{1}}^{1} \\
I_{N_{1}}^{t h}+G \cdot C S I(t) e l s e
\end{array} \quad \text { with } \quad G=\frac{I_{N_{r}}^{t h}-I_{N_{1}}^{t h}}{\operatorname{CSI}\left(f t_{N_{r}}^{1}\right)-\operatorname{CSI}\left(f t_{N_{1}}^{1}\right)}\right.
$$

To identify $G$, the rheobase currents of the first and last identified MNs $I_{N_{1}}^{t h}$ and $I_{N_{r}}^{t h}$ were estimated from a typical distribution of rheobase in a MN pool $I^{\text {th }}(j)=k_{1} \cdot \Delta_{\mathrm{I}}^{\left(\frac{j}{N}\right)^{k_{3}}}$, obtained as for the distribution of $F^{\text {th }}$, from normalized experimental data from populations of hindlimb alpha-MNs in adult rats and cats in vivo (Kernell, 1966; Fleshman et al., 1981; Kernell \& Monster, 1981; Gustafsson \& Pinter, 1984; Zengel et al., 1985; Foehring et al., 1987; Bakels \& Kernell, 1993; Gardiner, 1993; Krawitz et al., 2001). A $\Delta_{I}=9.1$-fold range in $\mathrm{MN}$ rheobase in $[3.9 ; 35.5] n A$ was taken (Manuel et al., 2019; Caillet et al., 2021), while a larger $\Delta_{I}$ is also consistent with the literature (Caillet et al., 2021, Table 4) and larger values of $I^{\text {th }}$ can be expected for humans (Manuel et al., 2019). 


\section{Step (3): LIF model - parameter tuning and distribution of electrophysiological properties in the}

\section{MN pool}

In the third step of the approach in Figure 1, LIF MN models are calibrated to mimic the discharge behaviour of the $N_{r}$ experimentally identified MNs.

\section{$\underline{\text { MN LIF model: description }}$}

A variant of the LIF MN model was chosen in this study for its relative mathematical simplicity and low computational cost and its adequacy in mimicking the firing behaviour of MNs (Teeter et al., 2018). LIF models describe the discharge behaviour of a $\mathrm{MN}$ of rheobase $I^{\text {th }}$ and input resistance $R$ as a capacitor charging with a time constant $\tau$ and instantaneously discharging at time $f t$ when the membrane voltage $V_{m}$ meets the voltage threshold $V_{t h}$, after which $V_{m}$ is reset and maintained to the membrane resting potential $V_{\text {rest }}$ for a time duration called 'inert period' IP in this study. For simplicity without loss of generalisation, the relative voltage threshold was defined as $\Delta V_{t h}=V_{t h}-V_{\text {rest }}>0$, and $V_{\text {rest }}$ was set to 0 .

The model is described by the following set of equations:

$$
\left\{\begin{array}{c}
\tau \frac{d V_{m}}{d t}=R \cdot I(t)-V_{m} \\
\tau=R C \\
V_{m}(f t)=\Delta V_{t h} \\
\lim _{t \rightarrow f t^{+}} V_{m}=0 \\
\forall t \in[f t ; f t+I P], V_{m}=0
\end{array}\right.
$$

The differential equation was solved with a time step $d t \leq 0.001 \mathrm{~s}$ as:

$$
\left\{\begin{array}{c}
V_{m}(0)=0 \\
\forall n \in \mathbb{N}^{*}, V_{m}[n T]=e^{-\frac{T}{\tau}} V_{m}[(n-1) T]+R \frac{T}{\tau} \cdot I(n T)
\end{array}\right.
$$

This model includes 5 electrophysiological parameters: $R, C, \tau, \Delta V_{t h}$ and $I P$. The parameters $R$ and $C$ were mathematically related to the $\mathrm{MN}$ surface area $\mathrm{S}$ as $R=\frac{k_{R}}{\mathrm{~S}^{2.4}}$ and $C=C_{m} \cdot S$ after an extensive meta-analysis of published experimental data on hindlimb alpha-MNs in adult cats in vivo (Caillet et al., 2021), that supports the equality $\tau=R C$ and the validity of Ohm's law in MNs as $I^{t h}=\frac{2.7 \cdot 10^{-2}}{R}=\frac{\Delta V_{t h}}{R}$, setting the constant value $\Delta V_{t h}=27 \mathrm{mV}$ in this study. The model was thus reduced to the MN size parameter $S$ and to the $I P$ parameter. The IP parameter is a phenomenological quantity that accounts for the $\mathrm{MN}$ absolute refractory period, the $\mathrm{MN}$ relative refractory period and reflects other phenomena not yet understood that define the inter-spike time intervals. 
LIF models can predict non-physiologically large values of MN firing frequency (FF) because of a linear to realistic ranges of values. Considering a constant supra-threshold current $I \gg I^{\text {th }}$ input to a LIF MN, the steady-state firing frequency FF predicted by the LIF model is:

$$
F F(I)=\frac{1}{I P-R C \ln \left(1-\frac{\Delta V_{t h}}{R I}\right)} \underset{I \gg I^{t h}}{\Longleftrightarrow} F F(I) \approx \frac{1}{I P+\frac{C \cdot \Delta V_{t h}}{I}}
$$

As $I$ and $C_{m}$ typically vary over a 10-fold and 2.4-fold range respectively (Caillet et al., 2021), the FF predicted by the LIF is dominantly determined by the value of $I P$ as the input current increasingly overcomes the MN current threshold: $F F(I) \approx \frac{1}{I P}$.

In the previous phenomenological models of MNs (Fuglevand et al., 1993; Callahan et al., 2013; Carriou et al., 2019), a maximum firing rate $\mathrm{FF}^{\mathrm{max}}$ was defined and a non-derivable transition from $F F(I)$ to constant $\mathrm{FF}^{\mathrm{max}}$ was set for increasing values of $I(t)$. Here, IP was integrated to the dynamics of the LIF model and was derived from experimental data to be MN-specific in the following manner. For each of the $N_{r}$ identified MNs, the time-course of the $\mathrm{MN}$ instantaneous discharge frequencies (IDFs) was first fit with a trendline $\left(I D F_{\text {trend }}\right)$ to neglect any unexplained random noise in the analysis. The mean $M$ of the trendline values during the plateau of force $\left[t_{r_{2}} ; t_{r_{3}}\right]$ was then obtained. If $\exists t \in$ $\left[t_{r_{1}} ; t_{r_{2}}-1\right], I D F_{\text {trend }}(t)>0.9 M$, i.e. if the $\mathrm{MN}$ reached during the ramp of $I(t)$ in $\left[t_{r_{1}} ; t_{r_{2}}-1\right]$ an IDF larger than $90 \%$ of the IDF reached one second before the plateau of force, the MN was identified to 'saturate'. Its $I P$ parameter was set to $I P=\frac{1}{\max \left(I D F_{\text {trend }}\right)}$, which constrains the MN maximum firing frequency for high input currents to $F F^{\max } \approx \frac{1}{I P}=\max \left(I D F_{\text {trend }}\right)$. A power trendline $I P(j)=a \cdot j^{b}$ was finally fitted to the pairs $\left(N_{i} ; I P_{N_{i}}\right)$ of saturating $\mathrm{MNs}$ and the $I P_{N_{i}}$ values of the non-saturating MNs were predicted from this trendline. To account for the residual variation in FF observed to remain at high $I(t)$ due to random electrophysiological mechanisms, the $I P$ parameter was randomized at each firing time, taking the value $I P+o$, where $o$ was randomly obtained from a normal gaussian distribution of $\frac{I P}{10}$ standard deviation.

\section{$\underline{\text { MN LIF model: MN size parameter calibration }}$}

The remaining unknown parameter - the MN size $S$ - defines the recruitment and firing dynamics of the LIF model. The size $S_{i}$ of the $i^{\text {th }}$ identified MN was calibrated by minimizing over the time range 
$\left[t_{t r_{0}} ; t_{t r_{3}}\right]$ (Table 1) the cost function $J\left(S_{i}\right)$ computed as the root-mean-square difference between experimental $F I D F_{i}^{\text {exp }}(t)$ and LIF-predicted $F I D F_{i}^{s i m}(t)$ filtered instantaneous discharge frequencies:

$$
\left\{\begin{array}{c}
J\left(S_{i}\right)=\sqrt{\frac{d t}{t_{r_{3}}-t_{r_{0}}} \cdot \sum_{t_{k}=t_{r_{0}}}^{t_{r_{3}}}\left(F I D F_{i}^{s i m}\left(t_{k}\right)-F I D F_{i}^{\exp }\left(t_{k}\right)\right)^{2}} \\
\min _{S_{i}} J\left(S_{i}\right), i \in \llbracket 1 ; N_{r} \rrbracket
\end{array}\right.
$$

To assess how well the calibrated LIF models can replicate the available experimental data, the normalized RMS error (nRMSE) (\%) and coefficient of determination $r^{2}$ between $\operatorname{FIDF}_{i}^{\exp }(t)$ and $F I D F_{i}^{s i m}(t)$, and the error in seconds between experimental and predicted recruitment times $\Delta f t_{i}^{1}=$ $f t_{i}^{1, \text { sim }}-f t_{i}^{1, \exp }$ were computed for the $N_{r}$ MNs. Finally, a power trendline $S(j)=k_{1} \cdot \Delta_{\mathrm{S}}^{\left(\frac{j}{N}\right)^{k_{3}}}$ was fitted to the pairs $\left(N_{i} ; S_{N_{i}}\right)$, and the continuous distribution of MN sizes in the entire pool of $N$ MNs was obtained. $\Delta_{S}=2.4$ was taken in Caillet et al. (2021). The $S(j)$ distribution defines the continuous distribution of the MN-specific electrophysiological properties across the MN pool (Caillet et al., 2021, Table 6).

\section{MN LIF model: parameter identification during the derecruitment phase}

The time-range $\left[t_{r_{3}} ; t_{r_{5}}\right]$ over which the MNs are being derecruited was not considered in the previous calibration. A linear trendline $I^{d t h}=k_{t h}^{d t h} \cdot I^{t h}$ was fitted to the association of experimental MN recruitment and recruitment rheobase of the $N_{r}$ recruited MNs. $k_{t h}^{d t h}<1$ was obtained, consistently with the literature, and suggests that MNs are derecruited at lower rheobase values than at recruitment. This was modelled by increasing the MN input resistance $R=\frac{k_{R}}{\mathrm{~S}^{2.4}}$ at derecruitment over the $\left[t_{r_{3}} ; t_{r_{5}}\right]$ time range as $k_{R}^{d}=\frac{k_{R}}{k_{t h}^{d t h}}>k_{R}$.

\section{Step (4): Simulating the MN pool firing behaviour}

The firing behaviour of the complete $\mathrm{MN}$ pool, i.e. the $\mathrm{MN}$-specific spike trains $s p_{j}(t)$ of the $N$ virtual MNs constituting the MN pool, was predicted with a cohort of $N$ LIF models receiving the common synaptic current $I(t)$ as input. The inert period $I P_{j}$ and $\mathrm{MN}$ size $S_{j}$ parameters scaling each LIF model were obtained from the distributions $I P(j)$ and $S(j)$ previously derived at Step (3). 


\section{$\underline{\text { Validation }}$}

\section{Validation 1}

We assessed whether the $N_{r}$ experimental spike trains $s p_{i}^{\text {exp }}(t)$ were accurately predicted by this 4step approach (Figure 1). Steps (2) and (3) (Figure 1) were iteratively repeated with samples of $N_{r}-1$ spike trains, where one of the experimentally recorded MNs was not considered. At each $\mathrm{i}^{\text {th }}$ iteration of the validation, the $i^{t h}$ identified spike train $s p_{i}^{e x p}(t)$ was not used in the derivation of the synaptic current $I(t)$ (step 2 ) and in the reconstruction of the $\mathrm{MN}$ size and $I P$ distributions $S(j)$ and $I P(j)$ in the MN pool (step 3). As in Step (4), the FIDF $F_{i}^{\text {sim }}$ of the $i^{\text {th }} \mathrm{MN}$ was finally predicted with a LIF model, which was scaled with the parameters $S\left(N_{i}\right)$ and $I P\left(N_{i}\right)$ predicted from the $S(j)$ and $I P(j)$ distributions. For validation, $F I D F_{i}^{s i m}$ was compared to the experimental $F I D F_{i}^{\text {exp }}$ with calculation of $\Delta f t_{i}^{1}, r^{2}$ and nRMSE values. This validation was iteratively performed for all the $N_{r}$ identified $\mathrm{MN}$ spike trains.

\section{Validation 2}

We assessed whether the $N$ MN spike trains $s p_{j}^{s i m}(t)$, predicted in Step 4 for the entire MN pool from the $N_{r}$ identified trains $s p_{i}^{\text {exp }}(t)$, accurately predicted the effective neural drive $\left(\mathrm{eND}_{\mathrm{N}}\right)$ to the muscle. The eND $\mathrm{N}_{\mathrm{N}}$ was computed as the $[0 ; 4] \mathrm{Hz}$ low-pass filtered cumulative spike train of the $N$ predicted spike trains, $C S T_{N}=\sum_{j=1}^{N} s p_{j}^{s i m}(t)$. As suggested for isometric contractions (Farina \& Negro, 2015), the normalized $e N D_{N}$ was compared for validation against the normalized experimental force trace $\bar{F}(t)$ with calculation of the nRMSE and $r^{2}$ metrics. $\bar{F}(t)$ was also compared with nRMSE and $r^{2}$ to the normalized effective neural drive $e N D_{N_{r}}$ computed directly from the $N_{r}$ experimentally identified MN spike trains. The added value of the presented workflow (Steps 1 to 4 ) in predicting the neural drive to muscle was finally assessed by comparing the (nRMSE, $r^{2}$ ) pairs obtained for the $e N D_{N_{r}}$ and $e N D_{N}$ traces.

\section{Applications}

Sensitivity analysis - Specific capacitance $C_{m}$

The capacitance parameter $C$ in the LIF model is related to the MN size $S$ as $C=C_{m} \cdot S$ (Caillet et al., 2021). The specific capacitance $C_{m}$, which dominantly affects the firing rate properties of the LIF model during the ramp of increasing $I(t)$ over $\left[t_{t r_{1}} ; t_{t r_{2}}\right]$, is accepted to be constant across a MN pool (Gentet et al., 2000; Caillet et al., 2021) with a reference value $C_{m}=0.9 \mu \mathrm{F} \cdot \mathrm{cm}^{2}$. However, $C_{m}$ cannot be directly measured in experiments and a wide range $[0.8 ; 10.8] \mu \mathrm{F} \cdot \mathrm{cm}^{2}$ of $C_{m}$ values is found in the 
literature. The workflow (Figure 1) was used to identify the value of $C_{m}$ that most accurately described the electrophysiological properties of the investigated $\mathrm{MN}$ pool. We iteratively performed step (3) over $\left[t_{t r_{0}} ; t_{t r_{3}}\right]$ and calibrated the MN size parameters $S_{i}$ of the $N_{r} \mathrm{MNs}$ for $0.1 \mu \mathrm{F} \cdot \mathrm{cm}^{2}$ increments to the value of $C_{m}$ in $[0.5 ; 3] \mu F \cdot \mathrm{cm}^{2}$. For each incremental step in the value of $C_{m}$, the nRMSE and $r^{2}$ values, that were obtained after MN size calibration between experimental and predicted FIDFs for the $N_{r} \mathrm{MNs}$, were averaged, providing one $\left(\overline{\mathrm{nRMSE}}, \overline{r^{2}}\right)$ pair per $C_{m}$ value. The value of $C_{m}$ returning the lowest $J\left(C_{m}\right)=\frac{\frac{\overline{n R M S E}}{100}-\overline{r^{2}}}{2}$ was assumed to best represent the electrophysiological properties of the MN pool and was retained for deriving the $S(j)$ distribution and for scaling the $N$ LIF models in step (4).

\section{Insights on the MN behaviour at derecruitment}

As discussed by Heckman \& Enoka (2012), the derecruitment phase over the time-range $\left[t_{r_{3}} ; t_{r_{5}}\right]$ is associated with the activation of several complex and sometimes not understood phenomena, which lead the FF-I gain to decrease and the MNs to be derecruited at lower IDFs than at recruitment. We therefore investigated whether the proposed approach could account for these adaptations. Using $N_{r}$ $S_{i}-I P_{i}$-calibrated LIF models from Step (3), the $N_{r} F I D F_{i}^{s i m}$ traces were iteratively simulated over the time-range $\left[t_{r_{2}} ; t_{r_{5}}\right]$ for $0.1 \mu \mathrm{F} \cdot \mathrm{cm}^{2}$ incremental changes in the value of the membrane specific capacitance $C_{m}$. The $F I D F_{i}^{s i m}$ results were compared to the $F I D F_{i}^{\text {exp }}$ traces with nRMSE and $r^{2}$ values. As before, the value of $C_{m}$ returning the lowest $J\left(C_{m}\right)=\frac{\frac{\overline{n R M S E}}{100}-\overline{r^{2}}}{2}$ was retained and was renamed $C_{m}^{d}$. If $C_{m} \neq C_{m}^{d}$, the approach would capture a variation in MN membrane electrophysiological mechanisms between recruitment and derecruitment phases. In such case, the individual spike trains $s p_{i}^{s i m}(t)$ were predicted with $C_{m}$ over the $\left[t_{r_{0}} ; t_{r_{3}}\right.$ [ time range and $C_{m}^{d}$ over the $\left[t_{r_{3}} ; t_{r_{5}}\right]$ time range.

\section{MN-driven muscle model}

The $N$ MN-specific spike trains $s p_{j}^{s i m}(t)$ predicted in step (4) were input to a phenomenological muscle model to predict the whole muscle force trace $F_{N}^{s i m}$ in a forward simulation of muscle voluntary isometric contraction. As displayed in Figure 3 , the muscle model was built as $N$ in-parallel Hill-type models which were driven by the simulated spike trains $s p_{j}^{s i m}(t)$ and replicated the excitationcontraction coupling dynamics and the contraction dynamics of the $N$ MUs constituting the whole muscle. The MU excitation-contraction coupling dynamics were modelled after Hatze's equations (Hatze, 1977; Hatze, 1980) and a model of sarcomere dynamics (Zot \& Hasbun, 2016). In brief, the binary spike train $s p_{j}^{s i m}(t)$ defined for each $j^{\text {th }} \mathrm{MU}$ the trains of nerve and muscle fibre action 
potentials that drove the transients of calcium ion $\mathrm{Ca}^{2+}$ concentration in the $\mathrm{MU}$ sarcoplasm and the sarcomere dynamics, yielding the time-history of the $\mathrm{MU}$ active state $a_{j}(t)$. The $\mathrm{MU}$ contraction dynamics were reduced to a normalized force-length relationship that scaled nonlinearly with the MU active state (Lloyd \& Besier, 2003) and transformed $a_{j}(t)$ into a normalized MU force trace $\bar{f}_{j}(t)$. The experiments being performed at constant ankle joint $\left(100^{\circ}, 90^{\circ}\right.$ being ankle perpendicular to tibia) and muscle-tendon length, it was simplified, lacking additional experimental insights, that tendon and fascicle length both remained constant during the whole contractile event at optimal MU length $\overline{l_{o p t, J}}=1$. The dynamics of the passive muscle tissues and of the tendon and the fascicle force-velocity relationships were therefore neglected. Finally, the MU-specific forces $f_{j}(t)$ were derived with a typical muscle-specific distribution across the $\mathrm{MU}$ pool of the $\mathrm{MU}$ isometric tetanic forces $f_{i s o}^{\max }(j)$ (Andreassen \& Arendt-Nielsen, 1987; Van Cutsem, M. et al., 1997; Van Cutsem, Michaël et al., 1998). The whole muscle force was obtained as the linear summation of the MU forces $F_{N}^{\operatorname{sim}}(t)=\sum_{j} f_{j}(t)$.

To validate $F_{N}^{\operatorname{sim}}(t)$, the experimental muscle force $F^{\exp }(t)$ was first approximated from the experimental force trace $F(t)$, which was recorded at the foot with a force transducer (Del Vecchio et al., 2019). The transducer-ankle joint and ankle joint-tibialis anterior moment arms $L_{1}$ and $L_{2}$ were estimated using OpenSim (Seth et al., 2018) and a generic lower limb model (Rajagopal et al., 2016). Using the model muscle maximum isometric forces, it was then inferred the ratio $q$ of transducer force $F(t)$ that was taken at MVC by the non-TA muscles spanning the ankle joint in MVC conditions. The experimental muscle force was estimated as $F^{\exp }(t)=(1-q) \cdot \frac{L_{1}}{L_{2}} \cdot F(t)$ and was compared with calculation of normalized maximum error (nME), nRMSE and $r^{2}$ values against the muscle force $F_{N}^{s i m}(t)$ predicted by the MN-driven muscle model from the $N$ neural inputs.

The whole muscle force $F_{N_{r}}^{\text {sim }}(t)$ was also predicted using the $N_{r}$ experimental spike trains $s p_{i}^{\text {exp }}(t)$ as inputs to the same muscle model of $N_{r}$ in-parallel Hill-type models (Figure 3 ). In this case, each normalized $\mathrm{MU}$ force trace $\bar{f}_{J}(t)$ was scaled with the same $f_{i s o}^{\max }(j)$ distribution, however assuming the $N_{r}$ MNs to be evenly spread in the MN pool. $F_{N_{r}}^{\text {sim }}(t)$ was similarly compared to $F^{\exp }(t)$ with calculation of $n M E, n R M S E$ and $r^{2}$ values. To assess the added value of the step (1-4) approach in the modelling of MN-driven muscle models, the (nME, nRMSE, $r^{2}$ ) values obtained for the predicted $F_{N_{r}}^{\text {sim }}(t)$ and $F_{N}^{\text {sim }}(t)$ were compared. 


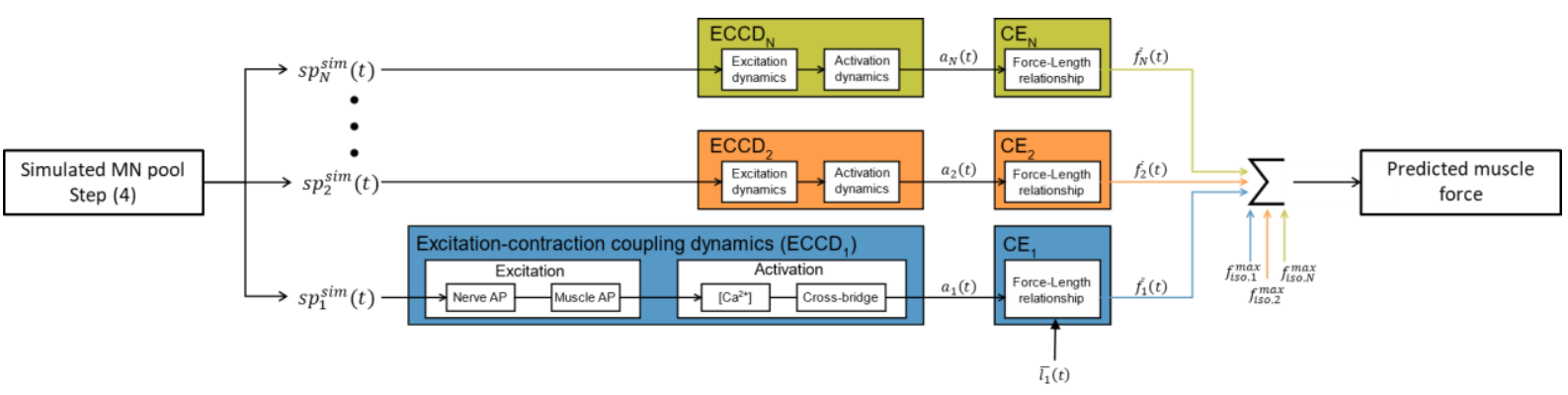

491 Figure 3: MN-driven neuromuscular model. The $N$ in-parallel Hill-type models take as inputs the $N$ spike trains $s p_{j}^{\text {sim }}(t)$ predicted in steps (1-4) and output the predicted whole muscle force trace $F_{m}^{s i m}(t)$. The MU-specific active states $a_{j}(t)$ are

493 obtained from the excitation-contraction coupling dynamics described as in (Hatze, 1980) and (Zot \& Hasbun, 2016). The MU normalized forces $\bar{f}_{J}(t)$ are computed by the $M U$ contractile elements $\left(C E_{j}\right)$ at $M U$ optimal length and are scaled with values of $\mathrm{MU}$ maximum isometric forces $f_{i s o, j}^{\max }$ to yield the $\mathrm{MU}$ force traces $f_{j}(t)$. The predicted whole muscle force is taken as $F_{N}^{s i m}(t)=\sum_{j} f_{j}(t)$. 


\section{RESULTS}

498

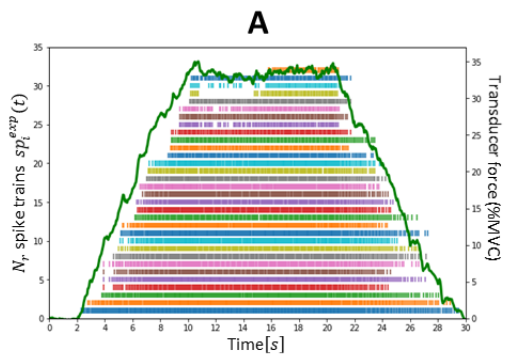

\section{Experimental data}

As reported in Table 1, the experimental datasets $D T A_{35}$ and $H T A_{35}$ respectively identified 32 and 21 spike trains $\operatorname{sp}_{i}^{\text {exp }}(t)$ from the trapezoidal isometric TA muscle contraction up to $35 \% \mathrm{MVC}, H T A_{50}$ identified $14 s p_{i}^{e x p}(t)$ up to $50 \% \mathrm{MVC}$, and $H G M_{30}$ identified $27 s p_{i}^{e x p}(t)$ from the GM muscle up to $30 \% \mathrm{MVC}$. The $N_{r}=32 \mathrm{MN}$ spike trains, identified in this dataset across the complete TA pool of $N=$ $400 \mathrm{MNs}$, are represented in Figure 4A in the order of increasing force recruitment thresholds $F_{i}^{\text {th }}$. The $N_{r}$ MNs were globally derecruited at relatively lower force thresholds (Figure 4B) and generally discharged at a relatively lower firing rate (Figure 4 C) at derecruitment than at recruitment.

508

509

510

511
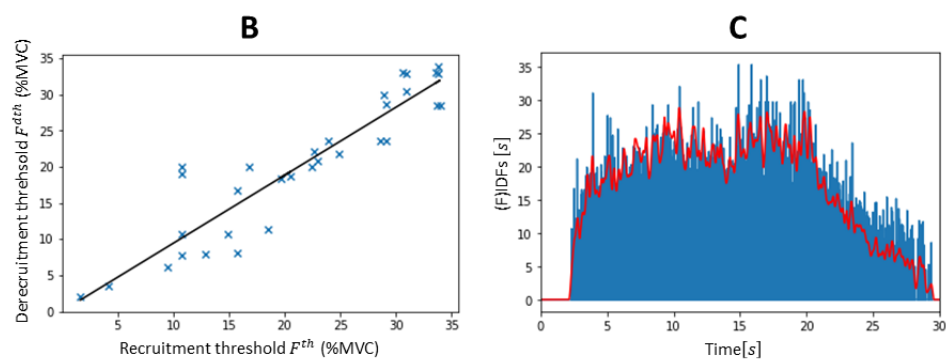

Figure 4: Experimental data obtained from HDEMG signal decomposition in the dataset DT $A_{35}$. (A) Time-histories of the transducer force trace in \%MVC (green curve) and of the $N_{r}=32 \mathrm{MN}$ spike trains identified from HDEMG decomposition and ranked from bottom to top in the order of increasing force recruitment thresholds $F^{\text {th }}$. (B) Association between force recruitment and derecruitment threshold, fitted by a linear trendline $y=0.9 \cdot x\left(r^{2}=0.85\right)$. (C) Time-histories of the instantaneous discharge frequencies (IDFs, blue trace) and of the moving-average filtered IDFs (FIDFs, red curve) of the lowestthreshold identified (1 $\left.{ }^{\text {st }}\right)$ MN.

\section{Step (1): MN mapping}

The $N_{r}=32 \mathrm{MNs}$ identified in the dataset $D T A_{35}$ were allocated to the entire pool of $N=400 \mathrm{MNs}$ according to their recruitment thresholds $F_{i}^{t h}$ (\%MVC). The typical TA-specific frequency distribution of the $\mathrm{MN}$ force recruitment thresholds $F^{\text {th }}$, which was obtained from the literature and reported in the bar plot in Figure 5A, was approximated (Figure 5B) by the continuous relationship $F^{\text {th }}(j)=0.50$. $\left(58.12 \cdot \frac{j}{N}+\Delta_{\mathrm{F}}^{\left(\frac{j}{N}\right)^{1.83}}\right), j \in \llbracket 1 ; N \rrbracket$. With this distribution, $231 \mathrm{TA} \mathrm{MNs}$, i.e. $58 \%$ of the MN pool is recruited below 20\%MVC, which is consistent with previous conclusions (Heckman \& Enoka, 2012).

From the $F^{\text {th }}(j)$ distribution, the $N_{r}$ identified MNs were mapped to the complete $\mathrm{MN}$ pool (blue crosses in Figure 5B) according to their recorded force recruitment thresholds $F_{i}^{\text {th }}$ (ordinates in Figure 
bioRxiv preprint doi: https://doi org/10.1101/2022.02.21 481337; this version posted February 22,2022 . The copyright holder for this preprint (which was not certified by peer review) is the author/funder, who has granted bioRxiv a license to display the preprint in perpetuity. It is made available under aCC-BY 4.0 International license.

4B). As shown in Figure 5C, the $N_{r}$ MNs identified experimentally were not homogeneously spread in the entire $\mathrm{MN}$ pool ranked in the order of increasing force recruitment thresholds, as two MNs fell in the first quarter of the MN pool, 5 in the second quarter, 18 in the third quarter and 5 in the fourth quarter. Such observation was similarly made in the three other experimental datasets, where no MN was identified in the first quarter and in the first half of the $\mathrm{MN}$ pool in the datasets $H G M_{30}$ and $H T A_{50}$ respectively (second column of Table 2). In all four datasets, mostly high-thresholds MNs were identified experimentally.
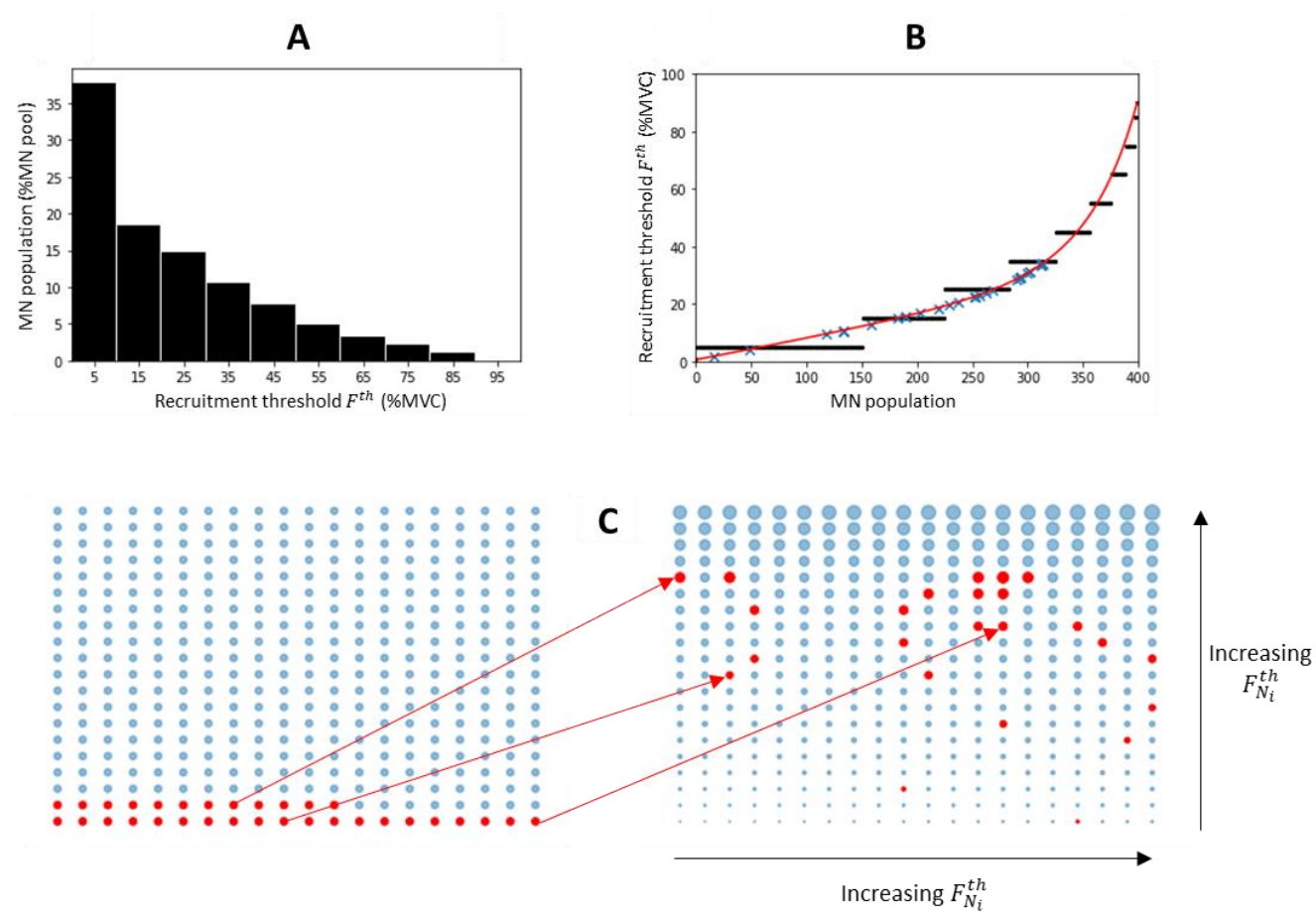

Figure 5: Distribution of force recruitment thresholds $F^{\text {th }}$ in the human Tibialis Anterior (TA) muscle and mapping of the $N_{r}$ identified MNs to the complete MN pool. (A) Typical partition obtained from the literature of the TA MN pool in 10\% increments in normalized $f F^{\text {th }}$. (B) Equivalent $F^{\text {th }}$ stepwise distribution (black dots) in a TA pool of $N=400 \mathrm{MNs}$, approximated by the continuous relationship $F^{\text {th }}(j)$ (red curve). The mapping (blue crosses) of the $N_{r}=32$ MNs identified in the dataset DT $A_{35}$ was obtained from the recorded $F_{i}^{\text {th }}$ (Figure $4 B$ ). (C) $N_{r}=32 \mathrm{MNs}$ (red dots) of unknown properties (Left) are mapped (Right) to the complete $M N$ pool from the $F^{\text {th }}(j)$ distribution, represented by the blue dots of increasing sizes. The MNs represented here are numbered from left to right and bottom to top from $j=1$ to 400 .

\section{Step (2): Common synaptic current $I(t)$}

To approximate the synaptic current $I(t)$ to the MN pool, the Cumulative Spike Train (CST) and the effective neural drive $(e N D)$ to the $\mathrm{MN}$ pool were obtained in Figure 6 from the $N_{r} \mathrm{MN}$ spike trains identified experimentally. After 20 random permutations of $\frac{N_{r}}{2}$ complementary populations of MNs, an average coherence of $\operatorname{coher}_{\frac{N_{r}}{2}}$,mean $=0.56$ was obtained between $\frac{N_{r}}{2}$ sized CSTs of the DTA $A_{35}$ dataset. From Figure 2 in Negro et al. (2016), a coherence of $\operatorname{coher}_{N_{r}}=0.8$ is therefore expected 
between the CST in Figure 6A and a typical CST obtained with another virtual group of $N_{r}=32 \mathrm{MNs}$, and by extension with the true CST obtained with the complete MN pool. The normalized $e N D$ and force trace (black and green curves respectively in Figure 6B) compared with $r^{2}=0.92$ and $n R M S E=$ $20.0 \%$. With this approach, we obtained $\operatorname{coher}_{N_{r}}>0.7, r^{2}>0.7$ and $n R M S E<30 \%$ for all four datasets, with the exception of $H G M_{30}$ for which $\operatorname{coher}_{N_{r}}<0.7$ (third column of Table 2). For the TA datasets, the sample $N_{r}=32 \mathrm{MNs}$ was therefore concluded to be sufficiently representative of the complete MN pool for its linearity property to apply, and the eND (red curve in Figure 6B for the dataset $\left.D T A_{35}\right)$ in the bandwidth $[0,10] \mathrm{Hz}$ was confidently identified to be the common synaptic input (CSI) to the MN pool. From Figure 2 in Negro et al. (2016), the computed CSI in Figure 6B (red curve) accounts for $60 \%$ of the variance of the true synaptic input, which is linearly transmitted by the $\mathrm{MN}$ pool, while the remaining variance is the MN-specific synaptic noise, which is assumed to be filtered by the $\mathrm{MN}$ pool and is neglected in the computation of the $e N D$ in this workflow.

A

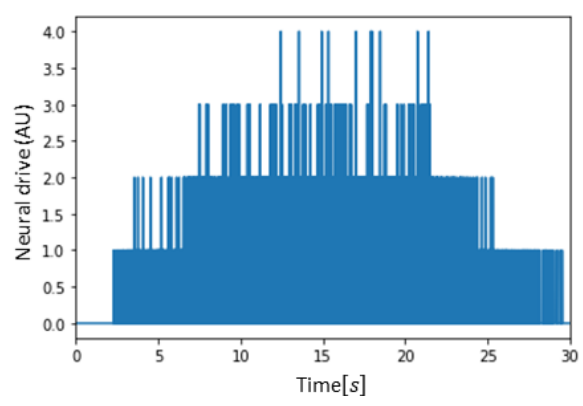

B

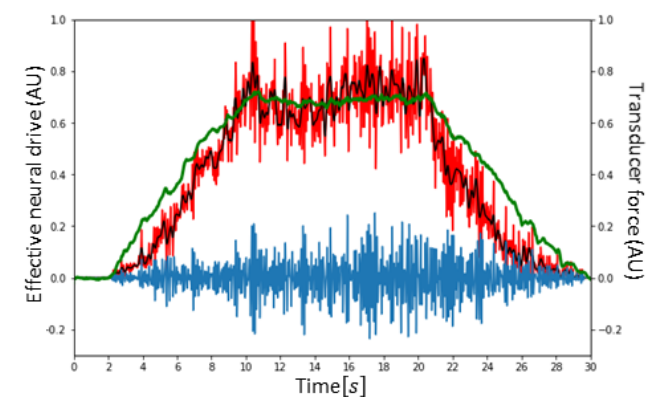

557

558

559

560

561

562

563

564

565

566

567

568

569

Figure 6: Neural drive to the muscle derived from the $N_{r}$ identified $M N$ spike trains in the dataset DT $A_{35}$. (A) Cumulative spike train (CST) computed by temporal binary summation of the $N_{r}$ identified $M N$ spike trains. (B) Effective neural driveUpon applicability of the linearity properties of subsets of the $\mathrm{MN}$ pool, the effective neural drive is assimilated as the synaptic input to the MN pool. The normalized common synaptic input (red), control (black) and noise (blue) are obtained from low-pass filtering the CST in the bandwidths relevant for muscle force generation. The normalized experimental force trace (green curve) is displayed for visual purposes.

To scale the normalized CSI (red curve in Figure 6B) to typical physiological values of synaptic current, the typical distribution of the $\mathrm{MN}$ membrane rheobase $I^{t h}(j)$ in a cat $\mathrm{MN}$ pool was obtained (Figure 7A) from the literature (Fleshman et al., 1981; Kernell \& Monster, 1981; Gustafsson \& Pinter, 1984; Zengel et al., 1985; Foehring et al., 1986; Munson et al., 1986; Foehring et al., 1987; Kernell \& Zwaagstra, 1989) as: $I^{\text {th }}(j)=3.9 \cdot 10^{-9} \cdot \Delta_{\mathrm{I}}^{\left(\frac{j}{N}\right)^{1.18}}$. From the normalized $C S I$ and the $I^{\text {th }}(j)$ distribution, the time-history of the common synaptic current $I(t)$ was obtained (Figure 7B):

$$
I(t)=\left\{\begin{array}{c}
0 \text { if } t<f t_{N_{1}}^{1} \\
3.9 \cdot 10^{-9}+6.0 \cdot 10^{-8} \cdot \operatorname{CSI}(t) \text { else }
\end{array}\right.
$$


A

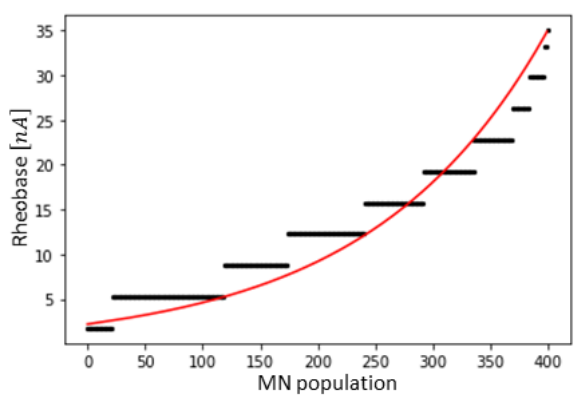

B

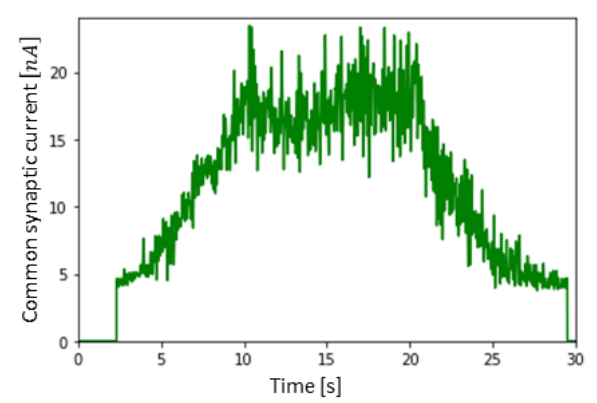

571

572

573

574

575

576

577

578

579

580

581

582

583

584

585

Figure 7: (A) Typical distribution of MN current recruitment threshold $I^{\text {th }}\left(N_{i}\right)$ in a cat $M N$ pool according to the literature.

(B) Common synaptic current $I(t)$ to the $M N$ pool, taken as a non-continuous linear transformation of the common synaptic input (red curve in Figure 6B) for the dataset DT $A_{35}$.

\section{Step (3): LIF model - MN size calibration and distribution}

Because of the modelling choices made for our MN LIF model, the MN inert period $(I P)$ and the MN size $S$ parameters entirely define the LIF-predictions of the MN firing behaviour. The $I P_{i}$ parameters of the $N_{r} \mathrm{MNs}$ in the dataset $D T A_{35}$ (Figure 8) were obtained from the maximum firing frequency of the 20 MNs identified to 'saturate', from which the distribution of $I P$ values in the entire pool of $N$ MNs was obtained: $I P(j)[s]=0.04 \cdot j^{0.05}$. With this approach, the maximum firing rate assigned to the first recruited and unidentified $M N$ is $\frac{1}{I P_{1}}=\frac{1}{0.04 \mathrm{~s}}=25 \mathrm{~Hz}$. The $I P$ distributions obtained with this approach for the three other datasets are reported in the fourth column of Table 2 and yielded physiological approximations of the maximum firing rate for the unidentified lowest -threshold MN for all datasets, with the exception of the dataset $H T A_{50}$, which lacks the information of too large a fraction of the MN pool for accurate extrapolations to be performed.

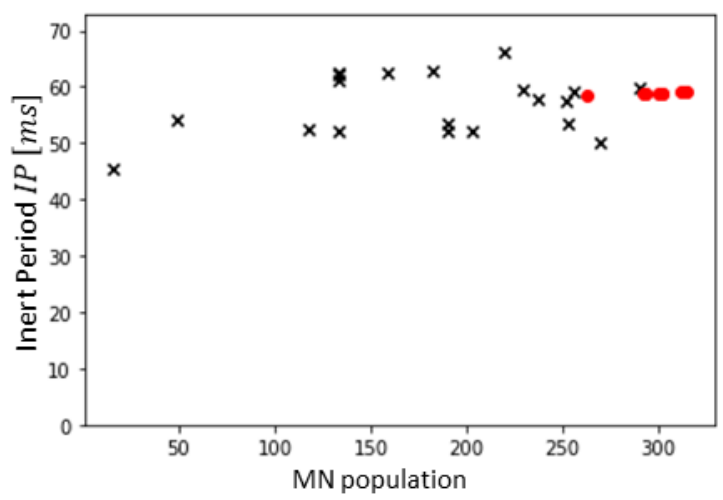

Figure 8: MN Inert Periods (IPS) in ms obtained from the experimental measurements of IDFs in dataset DT $A_{35}$. The twenty lowest-threshold MNs are observed to 'saturate' as described in the Methods and their IP (black crosses) is calculated as the inverse of the maximum of the trendline fitting the time-histories of their instantaneous firing frequency. The IPs of the 12 highest-threshold MNs (red dots) are obtained by trendline extrapolation. 
590

591

592

593

594

595

596

597

598

599

600

601

602

603

604

605

606

607

608

609

610

611

The size parameter $S_{i}$ of the $N_{r}$ LIF models was calibrated so that the LIF-predicted filtered discharge frequencies $F D I F_{i}^{s i m}(t)$ of the $N_{r}$ MNs replicated the experimental $F D I F_{i}^{e x p}(t)$, displayed in Figure 9A and B (blue curves). As shown in Figure 9C, the recruitment time $f t^{1}$ of two thirds of the $N_{r}$ identified MNs was predicted with an error less than $250 \mathrm{~ms}$. The calibrated LIF models were also able to accurately mimic the firing behaviour of the 27 lowest-threshold MNs as experimental and LIFpredicted FIDF traces compared with $r^{2}>0.8$ and $n R M S E<15 \%$ (Figure 9D and E). The scaled LIF models reproduced the firing behaviour of the five highest-threshold MNs $(\mathrm{Ni}>300)$ with moderate accuracy, with $\Delta f t^{1}, n R M S E$ values up to $-1.5 \mathrm{~s}, 18.2 \%$ and as low as $r^{2}=0.61$. The global results in Figure 9 confirm that the two-parameters calibrated LIF models can accurately reproduce the firing and recruitment behaviour of the $N_{r}$ experimental MNs. It must be noted that the $\Delta f t^{1}$ and $r^{2}$ metrics were not included in the calibration procedure and the $\left(\Delta f t^{1}, r^{2}\right)$ values reported in Figure 9 were therefore blindly predicted.
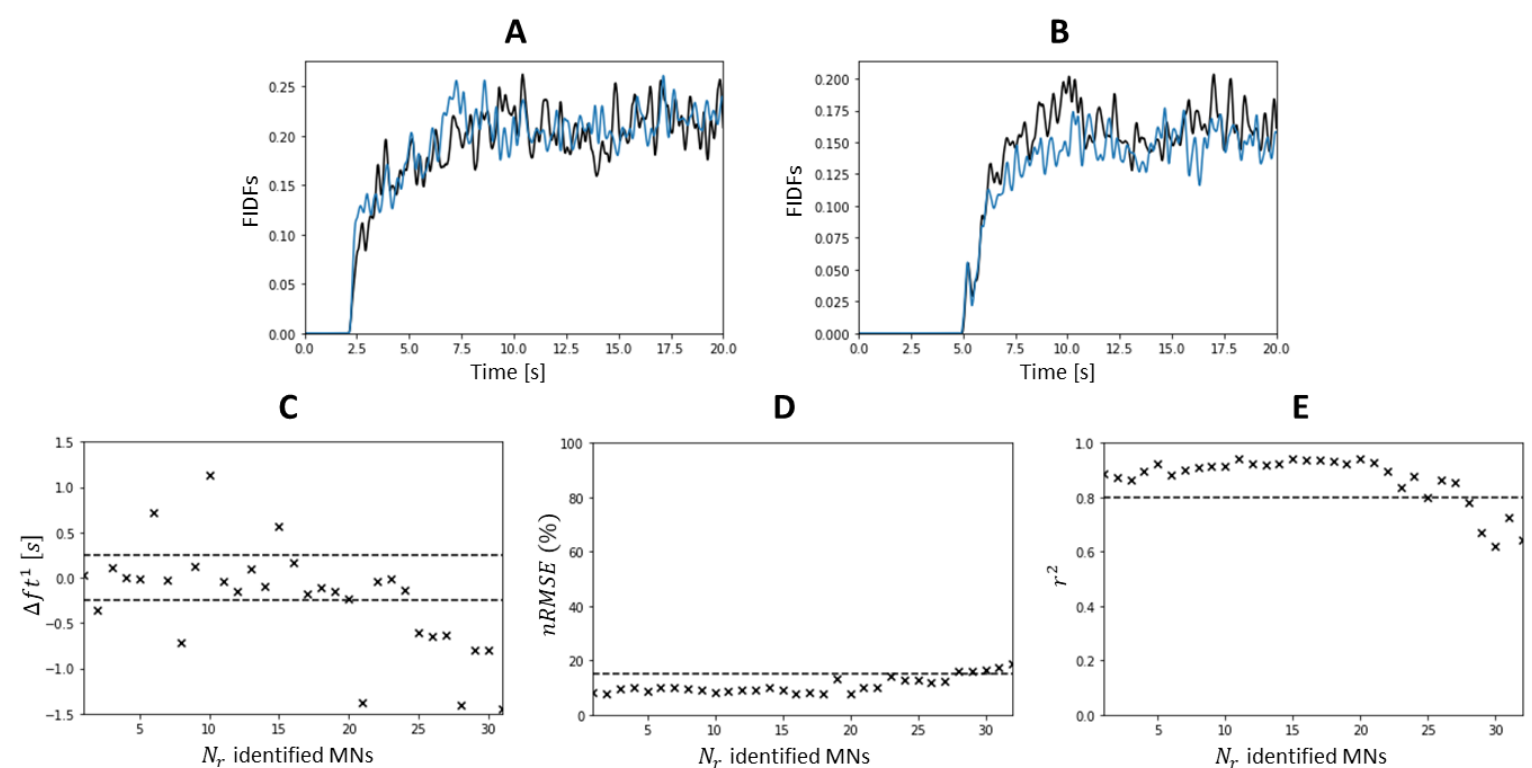

Figure 9: Calibration of the MN size $S_{i}$ parameter. (A and B) Time-histories of the experimental (black) versus LIF-predicted (blue) filtered instantaneous discharge frequencies (FIDFs) of the $1^{\text {st }}(A)$ and $11^{\text {th }}(B) M N$ s identified in the DT $A_{35}$ dataset after parameter calibration. (C) Absolute error $\Delta f t^{1}$ in seconds in predicting the $M N$ recruitment time with the calibrated LIF models. The accuracy of LIF-predicted FIDFs is assessed for each MN with calculation of the nRMSE (D) and $r^{2}$ (E) values. The dashed lines represent the $\Delta f t^{1} \in[-250 ; 250] m s, n R M S E \in[0 ; 15] \% M V C$ and $r^{2} \in[0.8,1.0]$ intervals of interest respectively.

The cloud of $N_{r}$ pairs $\left\{N_{i} ; S_{N_{i}}\right\}$ of data points (black crosses in Figure 10A), obtained from MN mapping (Figure $5 \mathrm{~B}$ and $\mathrm{C}$ ) and size calibration for the dataset $D T A_{35}$, were least-squares fitted (red curve in Figure $10 \mathrm{~A}, r^{2}=0.96$ ) by the power relationship, with $\Delta_{S}=2.4$ : 


$$
S(j)=1.14 \cdot 10^{-7} \cdot \Delta_{\mathrm{S}}^{\left(\frac{j}{N}\right)^{1.12}}
$$

As reported in the last column of Table 2, the minimum MN size (in $\mathrm{m}^{2}$ ) obtained by extrapolation of this trendline was in the range $[1.14,1.30] \cdot 10^{-1} \mathrm{~mm}^{2}$ for the datasets $D T A_{35}, H T A_{35}$ and $H G M_{30}$, which is consistent with typical cat data (Caillet et al., 2021), and the distribution of the MN sizes followed a more-than-linear and less-than-quadratic spline in these datasets. The dataset $H T A_{50}$ returned different results with a less-than-linear distribution of MN size and a low value for the MN size of the lowest-threshold MN in the MN pool.

Table 2: Intermediary results obtained for the datasets DT $A_{35}, H T A_{35}, H T A_{50}$ and $H G M_{30}$ from the three first steps of the approach. For each dataset are reported (1) the locations in the complete pool of $N$ MNs of the lowest- $\left(N_{1}\right)$ and highestthreshold $\left(N_{N_{r}}\right) M N$ s identified experimentally, (2) the coher ${ }_{N_{r}}$ value between the experimental and virtual cumulative spike trains (CST), and the coefficients defining the distributions in the complete MN pool of (3) the inert period (IP) parameter and of (4) the MN size (S). For the TA and GM muscles, $N=400$ and $N=550$ respectively.

\begin{tabular}{|l|c|c|c|c|c|c|c|}
\hline & \multicolumn{2}{|c|}{ Identified population } & CST & \multicolumn{2}{|c|}{$I P(j)[s]=a \cdot j^{b}$} & \multicolumn{2}{|c|}{$S(j)\left[m^{2}\right]=S_{\min } \cdot \Delta_{\mathrm{S}}^{\left(\frac{j}{N}\right)^{c}}$} \\
\hline Dataset & $N_{r}$ & $i \rightarrow N_{i}$ & coher $_{N_{r}}$ & $a$ & $b$ & $S_{\min }\left[m^{2}\right]$ & $c$ \\
\hline DT $_{35}$ & 32 & $16-314$ & 0.80 & 0.04 & 0.06 & $1.14 \cdot 10^{-7}$ & 1.12 \\
\hline$H T A_{35}$ & 21 & $77-313$ & 0.71 & 0.04 & 0.05 & $1.26 \cdot 10^{-7}$ & 1.34 \\
\hline$H T A_{50}$ & 14 & $232-346$ & 0.71 & 0.0006 & 0.80 & $1.09 \cdot 10^{-7}$ & 0.69 \\
\hline$H G M_{30}$ & 27 & $162-412$ & 0.65 & 0.03 & 0.20 & $1.30 \cdot 10^{-7}$ & 1.48 \\
\hline
\end{tabular}

As displayed in Figure $10 \mathrm{~B}$ for the dataset $D T A_{35}$, the $S(j)$ distribution determined the $\mathrm{MN}$-specific electrophysiological parameters (input resistance $R$ and membrane capacitance $C$ ) of a cohort of $N=$ $400 \mathrm{LIF}$ models, which predicted from $I(t)$ the spike trains of the entire pool of $N$ MNs (Figure 10C). 


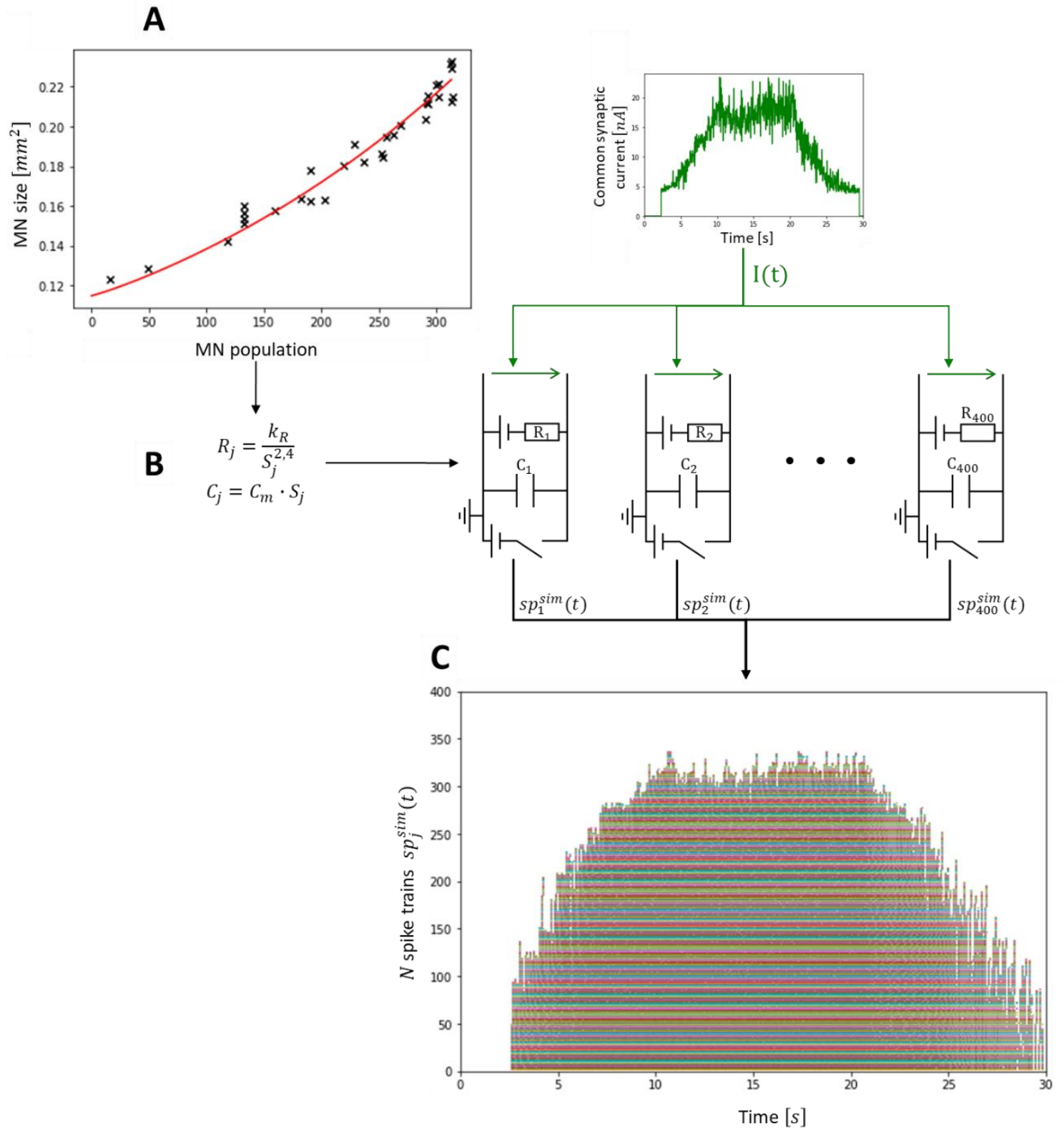
$M N$ sizes (black crosses) are lest-squares fitted by the power trendline $S(j)\left[\mathrm{m}^{2}\right]=1.15 \cdot 10^{-7} \cdot \Delta_{S}^{\left(\frac{j}{N}\right)^{1.12}}$, which reconstructs the distributions of $M N$ sizes in the complete $M N$ pool. (B) The $S(j)$ distribution determines the $M N$-specific $R$ and $C$ parameters of a cohort of $N$ LIF models, which takes as input the common synaptic current $I(t)$ and predicts $(C)$ the spike trains $s p_{j}^{\text {sim }}(t)$ of the $N$ virtual MNs constituting the complete $M N$ pool. 

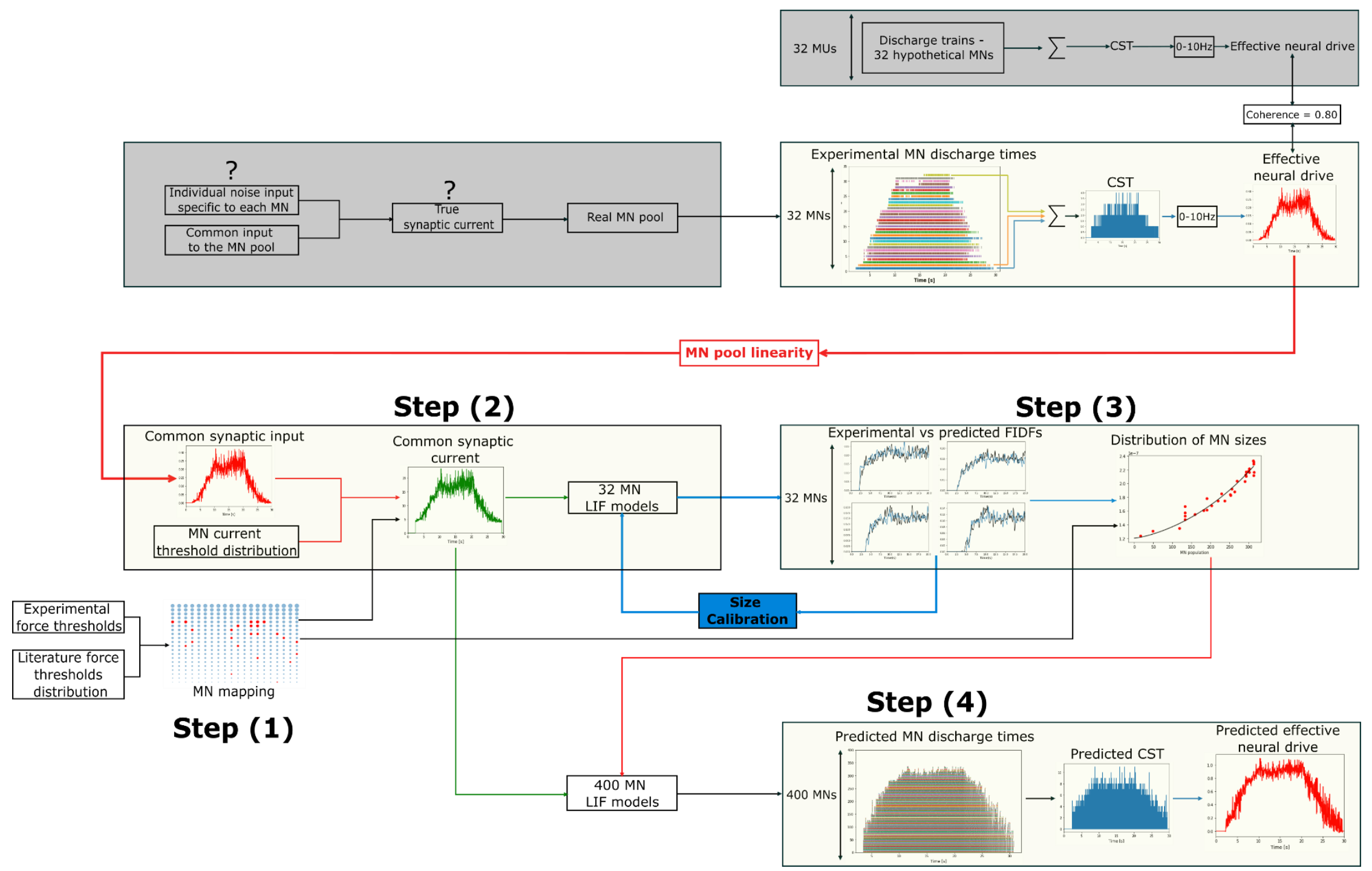

Figure 11: Detailed description of the 4-step workflow applied to the DT $A_{35}$ dataset. The firing activity of a fraction of the MN pool is obtained from decomposed HDEMG signals. These $N_{r}=$ 32 experimental spike trains provide an estimate of the effective neural drive to muscle and explain most of the $M N$ pool behaviour (coherence $=0.8$ ). From a mapping of the $N_{r}$ identified $M N$ s to the complete MN pool (Step (1)), literature knowledge on the typical distibution of $I^{\text {th }}$ in a mammalian $M N$ pool, and using the linearity properties of the population of $N_{r} M N s$, the common synaptic current $I(t)$ is estimated (Step (2)). I $(t)$ is input to $N_{r}$ LIF models of MN to derive, after a one-parameter calibration step minimizing the error between experimental and LIFpredicted FIDFs, the distribution of MN sizes across the complete MN pool (Step (3)). The distribution of MN sizes, which entirely describes the distribution of MN-specific electrophysiological parameters across the MN pool, scales a cohort of $N=400$ LIF models which transforms I $(t)$ into the simulated spike trains of the $N$ MNs of the MN pool (Step (4)). The effective neural drive to muscle is estimated from the $N$ simulated spike trains. 
644

645

646

647

648

649

650

651

652

653

654

655

656

657

658

659

660

661

662

663

664

665

666

667

668

669

670

671

\section{$\underline{\text { Validation }}$}

The four-step approach summarized in Figure 1 is detailed in Figure 11 and was validated in two ways.

\section{$\underline{\text { Validation } 1}$}

The simulated spike trains were validated for the $N_{r}$ MNs by comparing experimental and LIF-predicted FIDFs, where the experimental information of the investigated $M N$ was removed from the experimental dataset and not used in the derivation of the $I P(j)$ and $S(j)$ distributions of the synaptic current $I(t)$. For the $N_{r} \mathrm{MNs}$ of each dataset, Figure 12 reports the absolute error $\Delta f t^{1}$ in predicting the $\mathrm{MN}$ recruitment time ( $1^{\text {st }}$ row) and the comparison between experimental and LIF-predicted filtered instantaneous discharge frequencies (FIDFs) with calculation of $n R M S E$ (\%) (2 ${ }^{\text {nd }}$ row) and $r^{2}$ ( $3^{\text {rd }}$ row) values. In all datasets, the recruitment time of more than $60 \%$ of the $N_{r}$ identified MNs was predicted with an absolute error less than $\Delta f t^{1}=250 \mathrm{~ms}$. In all datasets, the LIF-predicted and experimental FIDFs of more than $80 \%$ of the $N_{r}$ MNs compared with $n R M S E<20 \%$ and $r^{2}>0.8$, while $70 \%$ of the $N_{r} \mathrm{MN}$ experimental and predicted FIDFs compared with $r^{2}>0.8$ in the dataset $H G M_{30}$. These results confirm that the four-step approach summarized in Figure 1 is valid for all four datasets for blindly predicting the recruitment time and the firing behaviour of the $N_{r}$ MNs recorded experimentally. The identified MNs that are representative of a large fraction of the complete MN pool, i.e. which are the only identified $\mathrm{MN}$ in the range $\left[N_{i}-\frac{N}{10} ; N_{i}+\frac{N}{10}\right]$ of the entire MN pool, such as the $1^{\text {st }} \mathrm{MN}$ in the dataset $D T A_{35}$ (Figure $5 \mathrm{C}$ ), are some of the $\mathrm{MNs}$ returning the highest $\Delta f t^{1}$ and $n R M S E$ and lowest $r^{2}$ values. As observed in Figure 12, ignoring the spike trains $s p_{i}^{\exp }(t)$ of those 'representative' MNs in the derivation of $I(t), I P(j)$ and $S(j)$ in steps (2) and (3) therefore affects the quality of the predictions more than ignoring the information of MNs that are representative of a small fraction of the MN population. In all datasets, $n R M S E>20 \%$ and $r^{2}<0.8$ was mainly obtained for the last-recruited MNs ( $4^{\text {th }}$ quarter of each plot in Figure 12) that exhibit recruitment thresholds close to the value of the synaptic current $I(t)$ during the plateau of constant force in the time range $\left[t_{t r_{2}} ; t_{t r_{3}}\right]$. In all datasets, the predictions obtained for all other MNs that have intermediate recruitment thresholds and are the most identified MNs in the datasets, were similar and the best among the pool of $N_{r}$ MNs. 

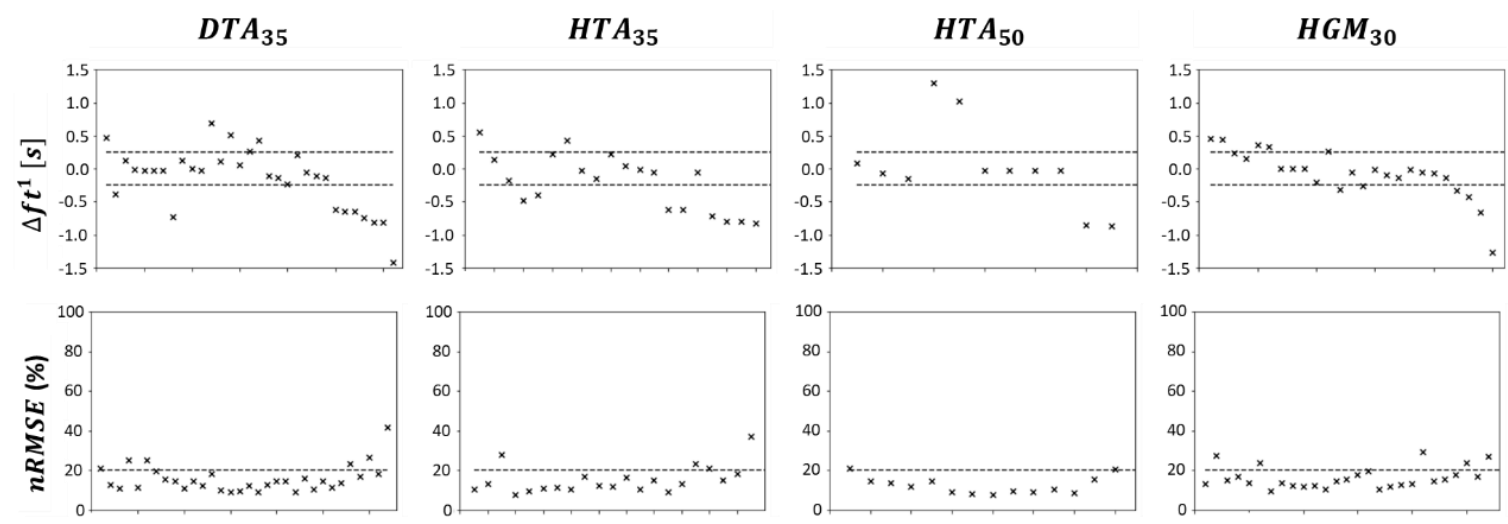

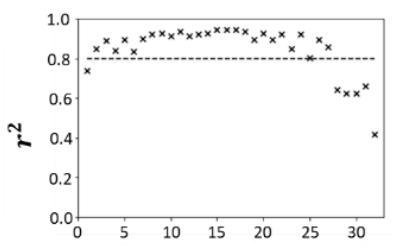

$N_{r}$ identified MNs

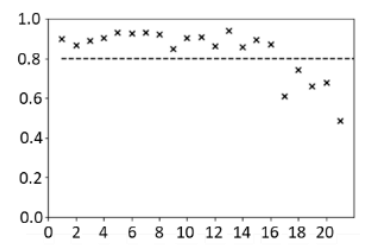

$N_{r}$ identified MNs

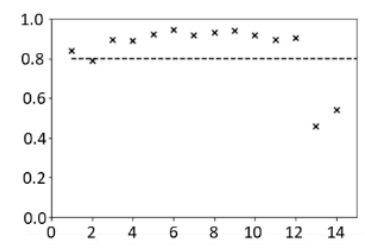

$N_{r}$ identified MNs

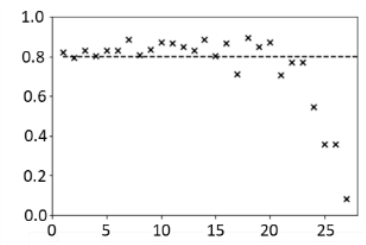

$N_{r}$ identified MNs
673

674

675

676

677

678

679

680

681

682

683

684

685

686

687

688

689

690

691

692

693

694

695

Figure 12: Validation of the $M N$ recruitment and firing behaviour predicted with the 4-step approach (Figure 1) for the $N_{r}$ MNs experimentally identified in the four datasets DT $A_{35}, H T A_{35}, H T A_{50}$ and $H G M_{30}$ described in Table 1. For the validation of each $i^{\text {th }}$ predicted MN spike train, the experimental information of the $i^{\text {th }}$ identified MN was omitted when deriving the current input $I(t)$ and the $I P(j)$ and $S(j)$ distributions (steps 2 and 3 ), from which the $I P_{i}$ and $S_{i}$ parameters are obtained without bias for the $i^{\text {th }} M N$. The spike train $\operatorname{sp}_{i}^{\text {sim }}(t)$ of the $i^{\text {th }} M N$ is then predicted with an IP $P_{i}, S_{i}$-scaled LIF model receiving $I(t)$ as input. The absolute error in predicting the experimental recruitment time $\Delta f t_{i}^{1}(s)$ is reported for each of the $N_{r}$ MNs in the first row of the figure. Experimental and filtered instantaneous discharge frequencies FID $F_{i}^{\text {exp }}(t)$ and $F I D F_{i}^{s i m}(t)$, computed from $s p_{i}^{\text {exp }}(t)$ and $s p_{i}^{\text {sim }}(t)$ are compared with calculation of $n R M S E(\%)$ and $r^{2}$ in the second and third rows of the figure. The dashed lines represent the $\Delta f t^{1} \in[-250 ; 250] m s, n R M S E \in[0 ; 15] \% M V C$ and $r^{2} \in[0.8,1.0]$ intervals of interest respectively.

\section{Validation 2}

The effective neural drive predicted by the 4-step approach summarized in Figure 1 was validated by comparing for isometric contractions, the normalized $e N D_{N}$ (orange traces in Figure 13) computed from the $N$ predicted spike trains $s p_{j}^{s i m}(t)$ to the normalized force trace $\bar{F}(t)$ (green trace in Figure 13). The $e N D_{N}$ was accurately predicted for the datasets $D T A_{35}$ and $H T A_{35}$, with $r^{2}=0.97$ and $n R M S E<8 \%$ (Table 3). The results obtained from the dataset $H G M_{30}$ returned $r^{2}=0.91$ and $n R M S E=12.5 \%$ (Table 3), accurately predicting the $e N D_{N}$ for the positive ramp and first half of the plateau of force and then underestimating the true neural drive $\left(H G M_{30}\right.$, Figure 13). The $e N D_{N}$ predicted for the dataset $H T A_{50}$ returned results of lower accuracy $\left(r^{2}=0.87\right.$ and $\left.n R M S E=16.0 \%\right)$ compared to the three other datasets. A null $e N D$ was predicted for one third of the simulation where a muscle force up to $20 \% M V C$ is generated, and the $e N D$ was overestimated for the rest of the (de)recruitment phase (HTA $A_{50}$, orange trace, Figure 12). As detailed in the discussion, the latter is explained by an inadequate $I P(j)$ distribution (Table 2 ), due to a lack of experimental information in 
696

697

698

699

700

701

702

703

704

705

706

707

708

709

710

711

712

713

714

the dataset $H T A_{50}$, which returns non-physiological maximum firing rates for the low-thresholds $\mathrm{MNs}$.

Two data points $(16 ; 0.032)$ and $(118 ; 0.037)$, obtained from the experimental data in the dataset $D T A_{35}$ with a scaling factor of $\frac{35}{50}$ applied to the $I P$ values, were appended to the list of $N_{r}$ data points $\left(N_{i} ; I P_{N_{i}}\right)$ to describe the maximum firing behaviour of the first half of the MN pool for which no MN was identified for the dataset $H T A_{50}$ (Table 2). A new $I P(j)$ distribution was obtained, returning an improved estimation of the $e N D_{N}\left(H T A_{50}\right.$, purple trace, Figure 12) with respectively lower and higher $n R M S E$ and $r^{2}$ metrics (Table 3). With three times lower $n R M S E$ and higher $r^{2}$ values for all four datasets (Table 3), the $e N D_{N}$ predicted from the 4-step approach (orange dotted traces in Figure 13) was a more accurate representation of the real effective neural drive than the $e N D_{N_{r}}$ (blue dotted traces in Figure 13) computed from the $N_{r}$ experimental spike trains, especially in the phases of $\mathrm{MN}$ (de)recruitment where the real effective neural drive was underestimated by $e N D_{N_{r}}$. The predicted $e N D_{N}$ also produced less noise than the $e N D_{N_{r}}$ trace during the plateau of force. With $r^{2}>0.85$ and $n R M S E<20 \%$, this four-step approach is valid for accurately reconstructing the $e N D$ to a muscle produced by a collection of $N$ simulated MN spike trains, which were predicted from a sample of $N_{r}$ experimental spike trains.

Table 3: For all four datasets, the $r^{2}$ and $n R M S E$ values obtained for the comparison of the time-histories of the normalized experimental force trace against the effective neural drive (1) eN $D_{N_{r}}$ and (2) eN $D_{N}$ computed from the spike trains of (1) the $N_{r}$ identified MNs and (2) the $N$ virtual MNs. The results for $H T A_{50}$ (1) were obtained with the standard approach, while those for $H_{T} A_{50}(2)$ were obtained with a revisited IP(j) distribution (see text).

\begin{tabular}{|c|c|c|c|c|}
\hline & \multicolumn{2}{|c|}{$N_{r}$ MNs (experimental) } & \multicolumn{2}{|c|}{$N$ MNs (simulated) } \\
\hline Dataset & $r^{2}$ & nRMSE (\%) & $r^{2}$ & nRMSE (\%) \\
\hline $\mathrm{DTA}_{35}$ & 0.92 & 20.0 & 0.97 & 7.5 \\
\hline $\mathrm{HTA}_{35}$ & 0.85 & 27.3 & 0.97 & 6.9 \\
\hline$H T A_{50}(1)$ & \multirow{2}{*}{0.83} & \multirow{2}{*}{26.7} & 0.87 & 16.0 \\
\hline$H T A_{50}(2)$ & & & 0.91 & 11.3 \\
\hline$H G M_{30}$ & 0.85 & 28.9 & 0.91 & 12.5 \\
\hline
\end{tabular}



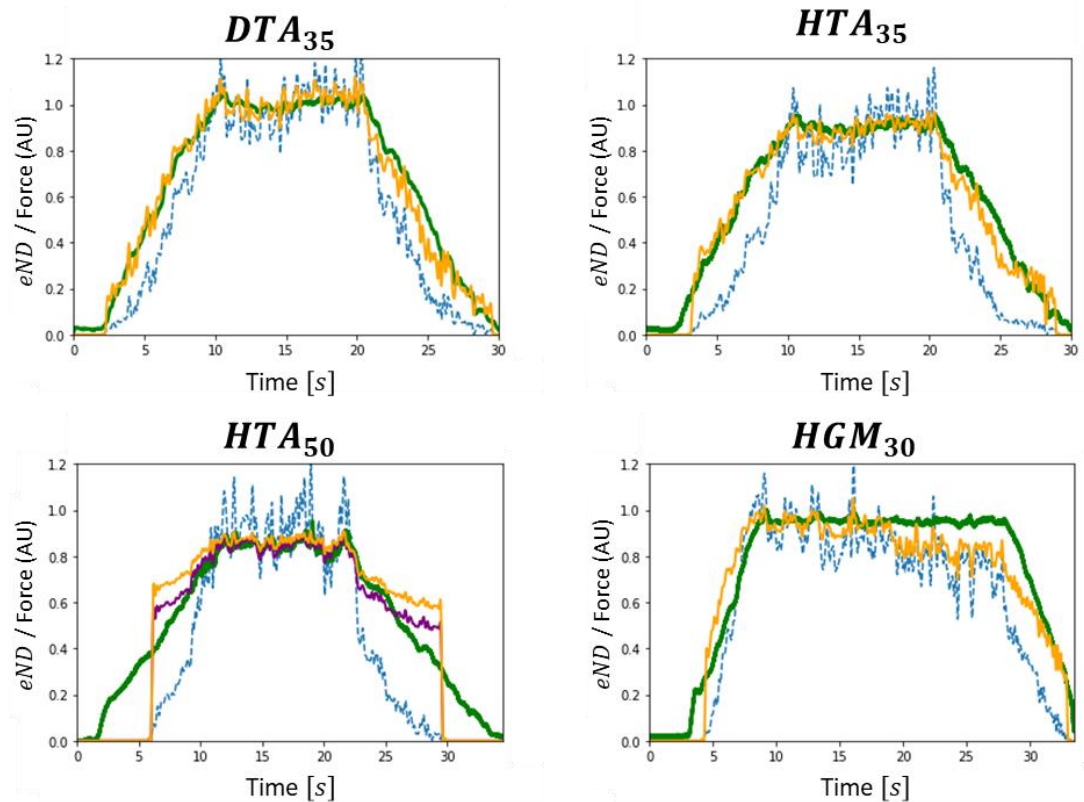

Figure 13: For the 4 datasets, validation against the normalized force trace $\bar{F}(t)$ (green trace) of the normalized effective neural drive to muscle ( $e N D_{N}$ ) produced by the complete $M N$ pool (orange trace) and computed from the $N M N$ spike trains $s p_{j}^{s i m}(t)$ predicted with the 4-step approach. For comparison, the $e N D_{N_{r}}$ directly computed from the $N_{r}$ identified $M N s$ is also reported (blue dashed trace). For the dataset $H T A_{50}$, an additional prediction was performed (purple trace), where the $I P(j)$ distribution obtained from step (3) was updated to return physiological maximum firing rates for all $N$ MNs.

\section{Applications}

Assessing the value of the specific capacitance $C_{m}$

After the parameter $S_{i}$ was calibrated in step (3), the 2-parameters LIF models most accurately replicated the firing behaviour of the $N_{r}$ identified MNs for a specific value of the MN membrane capacitance $C_{m}$. Figure 14 reports for the dataset $D T A_{35}$ the average among the $N_{r}$ MNs of the $n R M S E$ and $r^{2}$ values obtained between experimental and LIF-predicted FIDFs for incremental values of $C_{m}$. As for the three other datasets, a parabolic behaviour was obtained for $J\left(C_{m}\right)=\frac{\overline{n R M S E}-\overline{r^{2}}}{2}$ and a global minimum was found for $C_{m}=1.2,1.3,0.9$ and $1.3 \mu \mathrm{F} \cdot \mathrm{cm}^{2}$ for the $D T A_{35}, H T A_{35}, H T A_{50}$ and $H G M_{30}$ datasets respectively. These values are consistent with most findings from the literature and were retained for the final simulations, for which the results are reported in Figure 12 and Figure 13. 
A

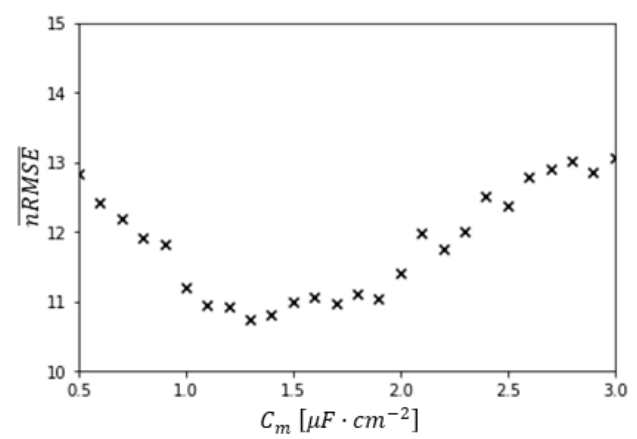

B

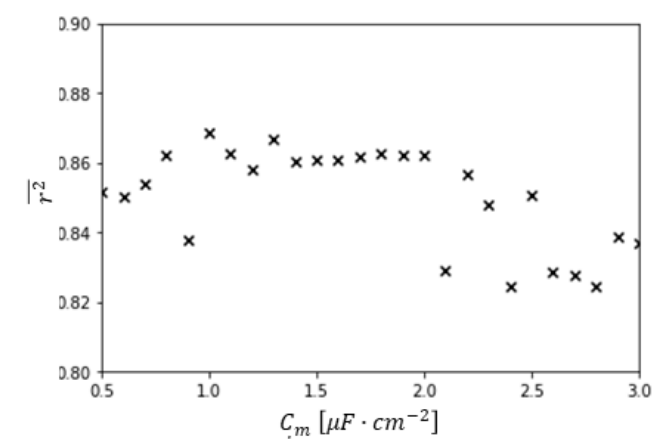

Figure 15: Sensitivity analysis on parameter $C_{m}^{d}$ for dataset DT $A_{35}$ over $\left[t_{r_{2}} ; t_{r_{5}}\right]$. The LIF-simulations were repeated for the $N_{r} M N s$ for $0.1 \mu \mathrm{F} \cdot \mathrm{cm}^{2}$ increments in the value of $C_{m}$. For each increment, the $n R M S E$ and $r^{2}$ values obtained between

Figure 14: Sensitivity analysis on parameter $C_{m}$ for the dataset DT $A_{35}$ over $\left[t_{r_{0}} ; t_{r_{3}}\right]$. The $M N$ size calibration of the $N_{r} M N s$ was repeated for $0.1 \mu \mathrm{F} \cdot \mathrm{cm}^{2}$ increments in the value of $C_{m}$. For each increment, the $n R M S E$ and $r^{2}$ values obtained for the $N_{r}$ calibrated MNs were averaged. The best $\left\{\overline{n R M S E}, \overline{r^{2}}\right\}=\{11 \%, 0.86\}$ pair was obtained for $C_{m}=1.2 \mu F \cdot \mathrm{cm}^{2}$ for the DT $A_{35}$ dataset.

\section{$\underline{\text { Specific capacitance } C_{m}^{d} \text { at derecruitment }}$}

Figure 15 reports for the dataset $D T A_{35}$ the sensitivity of the LIF-predicted $F I D F^{\operatorname{sim}}(t)$ time-histories to the value of the MN specific capacitance $C_{m}^{d}$ over the time-range $\left[t_{r_{2}} ; t_{r_{5}}\right]$ during which the MUs are being derecruited. In this analysis, the LIF models were scaled with the $S_{i}$ parameter calibrated in Step (3) and with the $C_{m}$ parameter value obtained from the sensitivity analysis in Figure 14. In all four datasets, $J\left(C_{m}\right)=\frac{\overline{n R M S E}-\overline{r^{2}}}{2}$ was again parabolic and a global minimum was found for $C_{m}^{d}=$ 1.8, 1.8, 1.2 and $2.2 \mu \mathrm{F} \cdot \mathrm{cm}^{2}$ for the datasets $D T A_{35}, H T A_{35}, H T A_{50}$ and $H G M_{30}$, respectively. In all four datasets, the MN specific capacitance increased during the derecruitment phase as $C_{m}^{d}=k \cdot C_{m}$ with $k \in[1.3 ; 1.7]$. These values for the parameter $C_{m}^{d}$ were retained for the final simulations (Figure 12 and Figure 13) over the $\left[t_{r_{3}} ; t_{r_{5}}\right]$ time range.
A

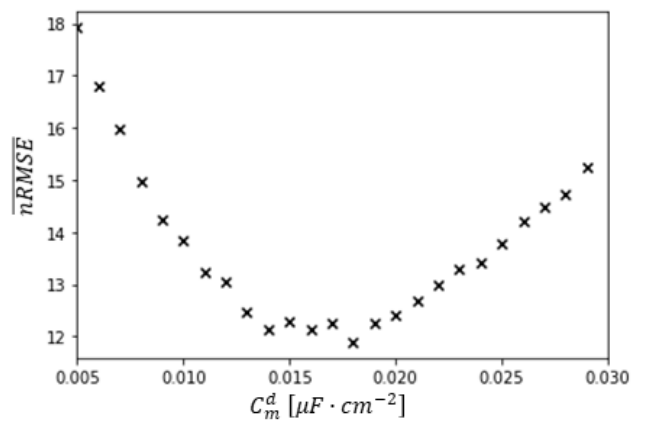

B

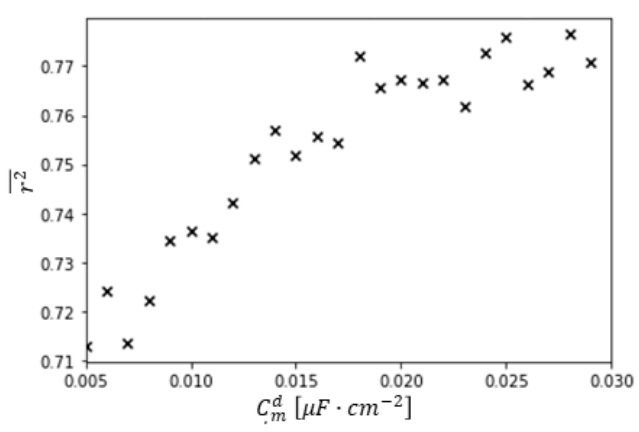
predicted and experimental FIDFs were averaged. The best $\left\{\overline{n R M S E}, \overline{r^{2}}\right\}=\{12 \%, 0.77\}$ pair was obtained for $C_{m}=$ $1.8 \mu \mathrm{F} \cdot \mathrm{cm}^{2}$ for the DT $A_{35}$ dataset. 


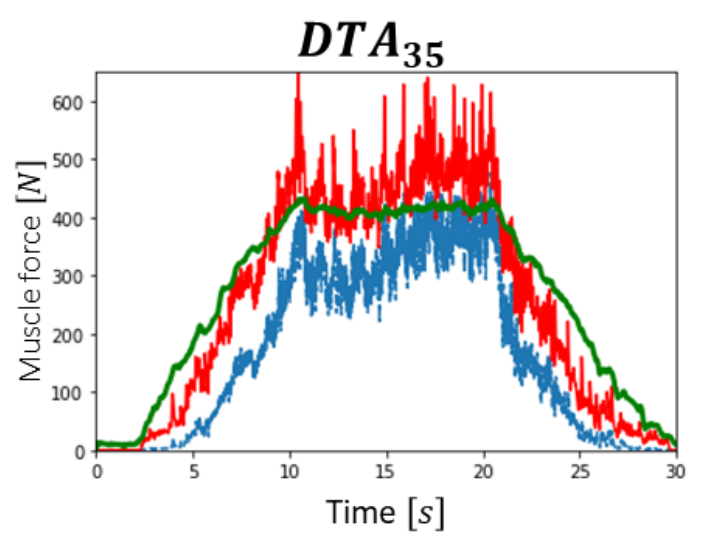

\section{MN-driven muscle model} Figure 3.

Figure 16 reports for the datasets $D T A_{35}$ and $H T A_{35}$, for which the $e N D_{N}$ was the most accurately predicted among the four datasets (Figure 13, Table 3), the time evolutions of the whole muscle force $F(t)$ recorded experimentally (green curve) and the whole muscle forces predicted with the MNdriven muscle model described in Figure 3 using $N_{r}$ experimental $\left(F_{N_{r}}(t)\right.$, blue curves) and $N$ simulated $\left(F_{N}(t)\right.$, red curves) spike trains as inputs. The muscle force trace $F_{N}(t)$ obtained from the $N$ simulated spike trains derived in steps (1-4) compared with the experimental force $F(t)$ with $r^{2}=0.91$ and $n R M S E=15 \%$ for the dataset $D T A_{35}$ and $r^{2}=0.91$ and $n R M S E=16 \%$ for the dataset $H T A_{35}$. These results validate the approach provided in this study for predicting the whole muscle force as a collection of MU force contributions from the MN-specific contributions to the effective neural drive. For the dataset $D T A_{35}$, a high noise in $F_{N}(t)$ was however predicted during the plateau of force, while the true muscle force was underestimated during the phases of $\mathrm{MN}$ (de)recruitment in both cases. The muscle force trace $F_{N}(t)$ obtained from the $N \mathrm{MN}$ spike trains returned more accurate predictions than $F_{N_{r}}(t)\left(r^{2}=0.84\right.$ and $n R M S E=27 \%$ for the dataset $D T A_{35}$ and $r^{2}=0.70$ and $n R M S E=$ $36 \%$ for the dataset $D T A_{35}$ ), in which case the reconstruction of the $\mathrm{MN}$ pool in steps (1-4) was disregarded and the $N_{r}$ experimental spike trains were directly used as inputs to the muscle model in 


\section{DISCUSSION}

775

This study reports a novel four-step approach, summarized in Figure 1 and displayed in detail in Figure 11 , to reconstruct the recruitment and firing behaviour of a complete human pool of $N$ MNs from a sample of $N_{r}$ experimental spike trains obtained from the decomposition of HDEMG or intramuscular recordings during voluntary contractions. This approach can help neuroscientists, experimentalists, and modelers to investigate $\mathrm{MN}$ pool neuromechanics, better understand experimental datasets, and control more detailed neuromuscular models to advance our understanding of the neural strategies taken by the human central nervous system to control voluntary muscle contractions.

The three first steps of our approach identify from a sample of $N_{r}$ experimental spike trains a distribution of the $\mathrm{MN}$ electrophysiological properties across the $\mathrm{MN}$ pool. The $N_{r} \mathrm{MN}$ spike trains are used to approximate the common synaptic drive $I(t)$ to the complete $\mathrm{MN}$ pool (Figure 7). $I(t)$ is input to a cohort of $N_{r}$ LIF models. The LIF firing behaviour is entirely described by the MN Inert Period parameter $I P_{i}$, derived from the experimental data (Figure 8), and by the MN size $S_{i}$ parameter to which all the MN electrophysiological properties are related, according to the relations provided by Caillet et al. (2021). After calibration of the $S_{i}$ parameter, the $N_{r}$ LIF models accurately mimic the filtered discharge behaviour and accurately predict the recruitment dynamics of the $N_{r}$ experimental MNs (Figure 9). The $N_{r} \mathrm{MNs}$ are allocated into the complete $\mathrm{MN}$ pool (Figure $5 \mathrm{~B}$ and $\mathrm{C}$ ) according to their recorded force recruitment thresholds $F_{i}^{\text {th }}$ (Figure 4B) and a typical species- and muscle-specific $F^{t h}(j)$ distribution (Figure 5). From the previous findings, the continuous distribution of $\mathrm{MN}$ sizes $S(j)$ (Figure 10A) is derived for the complete pool of $N$ MNs. $S(j)$ defines the electrophysiological properties (Caillet et al., 2021) of the MNs constituting the complete MN pool. The neural behaviour of the complete pool of $N$ MNs is predicted in the $4^{\text {th }}$ step with a cohort of $N S_{j}-I P_{j}$-scaled LIF models and the application of the common synaptic drive $I(t)$ (Figure 10).

\section{Validation of the approach}

The approach was successfully validated both for individual MNs and for the complete pool of $N$ MNs. By blindly scaling the LIF models in steps (1-3) with permutations of $N_{r}-1$ input experimental spike trains, the filtered discharge behaviour and the recruitment dynamics of the $N_{r}$ individual MNs were accurately predicted for the four investigated datasets (Figure 12). The effective neural drive $\left(e N D_{N}\right)$ to muscle elicited by the complete pool of $N$ MNs was also accurately predicted by the 4-step approach for the $H T A_{35}, D T A_{35}$ and $H G M_{30}$ datasets (Figure 13, Table 3). The latter result suggests that the 
recruitment dynamics and the normalized distribution of firing rates across the firing $\mathrm{MN}$ pool were accurately predicted for the non-identified population of $N-N_{r}$ MNs.

The intermediary predictions output by the MN pool model are besides consistent with other findings from the literature and suggest that the four-step approach realistically describes the true MN pool neurophysiology and dynamics. For example, while the MN size parameter calibration (Figure 9) only relies on the minimization of the root-mean square difference between experimental and predicted FIDFs, the normalized time-history of the FIDFs (high $r^{2}$ in Figure 12) and the MN recruitment times (low $\Delta f t^{1}$ values in Figure 12) of the $N_{r}$ MNs are both accurately mimicked (Figure 9) and blindly predicted (Figure 12) for all four datasets. The $S(j)$ distribution obtained from the LIF calibrations in step (3) is besides consistent with the mathematical relationship existing between rheobase and MN size (Caillet et al., 2021), which was not used in the current approach. The distribution in rheobase (Figure 7A) $I^{\text {th }}\left(N_{i}\right)=3.9 \cdot 10^{-9} \cdot 9.1\left(\frac{N_{i}}{N}\right)^{1.18}=3.9 \cdot 10^{-9} \cdot\left(2.4\left(\frac{N_{i}}{N}\right)^{1.12}\right)^{2.5 \cdot\left(\frac{N_{i}}{N}\right)^{0.06}} \quad$ and of MN size $S\left(N_{i}\right)=$ $1.14 \cdot 10^{-7} \cdot 2.44^{\left(\frac{N_{i}}{N}\right)^{1.12}}$ (Figure 10A) yield $I^{\text {th }}\left(N_{i}\right)=3.9 \cdot 10^{-9} \cdot\left(\frac{S\left(N_{i}\right)}{1.14 \cdot 10^{-7}}\right)^{2.5 \cdot\left(\frac{N_{i}}{N}\right)^{0.06}}$. Considering that $\left(\frac{N_{i}}{N}\right)^{0.06} \in[0.7 ; 1.0]$, this relation respectively yields for the smallest and largest MNs $I_{1}^{\text {th }}=1.2 \cdot 10^{4}$. $S_{1}^{1.8}$ and $I_{400}^{\text {th }}=8.9 \cdot 10^{8} \cdot S_{400}^{2.5}$, which are consistent with the relationship $I_{t h}=3.8 \cdot 10^{8} \cdot S^{2.5}$ that was obtained from an extensive literature meta-analysis by Caillet et al. (2021). The range of MN surface areas $[0.15 ; 0.36] \mathrm{mm}^{2}$ and input resistances $[0.5 ; 4.1] \mathrm{M} \Omega$ obtained from the $S(j)$ distribution falls into the classic range of MN properties for cats (Manuel et al., 2019; Caillet et al., 2021), which is consistent with the distribution of rheobase values used in Step (2) that was obtained from cat data. The distribution of MN input resistance in the MN pool, obtained from the calibrated sizes $S(j)$, defines the individual MN rheobase thresholds and predicts that $85 \%$ of the human TA MU pool is recruited at $35 \% \mathrm{MVC}$ (Figure 10C), which is consistent with previous findings in the literature (Van Cutsem, M. et al., 1997; Heckman \& Enoka, 2012).

\section{Added value of the approach}

The workflow provides a method to support future experimental investigations and better understand datasets of $N_{r}$ experimental spike trains obtained from signal decomposition. HDEMG decomposition techniques commonly identify samples of few tens of MNs (Del Vecchio et al., 2020) at most, i.e. typically less than $10 \%$ of the MU pool. The signal decomposition process can be refined and more MNs can be identified by iteratively running this workflow and using the $N-N_{r}$ simulated spike trains of the non-identified MNs as a model for identifying more MNs from the recorded signals. The method identifies credible $\mathrm{MN}$-specific electrophysiological and morphometric properties, including $\mathrm{MN}$ 
membrane surface area, input resistance, capacitance, rheobase and time constant, for the dataset of $N_{r}$ experimental MNs and for the $N-N_{r}$ MNs that cannot be recorded. The method also provides two-parameter scaled LIF models described in step (3) to accurately replicate the recruitment and firing behaviour of the $N_{r}$ identified MNs (Figure 9). This method shows in Figure 5, Figure 10A and Table 2, that the $N_{r}$-sized samples of MNs currently identified from HDEMG decomposition are not representative of the complete $\mathrm{MN}$ pool, as demonstrated by the $[0.65 ; 0.80]$ coher $_{N_{r}}$ values in Table 2 , and provides the $e N D_{N}$ computed from the $N$ simulated $\mathrm{MN}$ spike trains of the complete MN population to tackle this limitation. EMG decomposition shows a bias towards identifying large highthreshold MUs because of their larger electric signal amplitude detected by the HDEMG electrodes. Consequently, the $\operatorname{CST}_{N_{r}}$ computed from the $N_{r}$ identified MNs is not an accurate representation of the true $e N D$ (Figure 13, Table 3). During the force ramps in $\left[t_{1} ; t_{2}\right]$ and $\left[t_{3} ; t_{4}\right]$ (Figure 2), where the $e N D_{N_{r}}$ can reach $70 \% \mathrm{nME}$, the $e N D_{N}$ is accurately estimated because it includes the firing activity of the complete fraction of the smaller MNs that were underrepresented in the experimental dataset. The $e N D_{N}$ besides includes the complete population of high-threshold MNs, which reduces noise (orange vs blue trace in Figure 13) during the plateau of force in $\left[t_{2} ; t_{3}\right]$. The $e N D_{N}$ also filters the local non-physiological variations displayed by the $e N D_{N_{r}}$ such as the decrease in $e N D_{N_{r}}$ in $[10 ; 15] s$ in $D T A_{35}$ and $H T A_{35}$ (top plots in Figure 13), the sudden rise and drop in $e N D_{N_{r}}$ at $t=10 \mathrm{~s}$ and $t=23 \mathrm{~s}$ in $H T A_{50}$ (bottom left plot in Figure 13) or the steeply decreasing $e N D_{N_{r}}$ from $t=28 s$ in $H G M_{30}$ (bottom right plot in Figure 13). Understanding these limitations has important implications in neuromuscular modelling when the $C S T_{N_{r}}$ is input to a muscle model as a unique neural control (Sartori 2017, Kapelner 2020, Thompson 2018).

As it relies on a physiologically realistic description of the MN neurophysiology, this validated method is a tool for testing neurophysiological hypotheses and investigating some mechanisms and properties of the complete MN pool that cannot be directly observed experimentally in conditions of human voluntary contractions. The sensitivity analysis in Figure 14 reveals that the membrane specific capacitance $C_{m}$, which cannot be measured in humans, equals to $1.2 \mu \mathrm{F} \cdot \mathrm{m}^{-2}$ in human hindlimb muscles, which is consistent with previous findings in mammals (Gentet et al., 2000). According to the sensitivity analysis in Figure 15, the mechanisms that lead the MNs to discharge at relatively lower firing rates at derecruitment than at recruitment (Figure 4C) can be explained by an increase of the $\mathrm{MN}$ membrane specific capacitance by a factor in $[1.3 ; 1.7]$ when $\mathrm{MNs}$ are derecruited. As the $S, C, R, \tau$ parameters of the LIF models cannot be directly measured in humans (Manuel et al., 2019) , and considering that the normalized mathematical relationships relating the $\mathrm{MN}$ electrophysiological parameters can be extrapolated to humans (Caillet et al., 2021), the four-step approach is an adequate 
mammals and humans and to estimate typical human-specific ranges of absolute values for these properties.

This four-step approach advances the state-of-the-art in MN pool modelling. As discussed in the introduction, using a sample of $N_{r}$ experimental $\mathrm{MN}$ spike trains permits for the first time in human $\mathrm{MN}$ pool modelling to (1) estimate the true time-course of the common synaptic input to the MN pool, which cannot be measured experimentally, (2) evaluate with a one-parameter calibration, available HDEMG data and knowledge from the literature, the MN-specific electrophysiological properties of all the MNs in the MN pool and a realistic distribution of these properties across the pool, which could, to date, only be obtained in specialized experimental and $\mathrm{MN}$ pool modelling studies in animals (Raikova et al., 2018), and (3) validate the forward predictions of MN spike trains and effective neural drive to muscle to human experimental data. The pool of LIF models, the maximum firing frequency of which is obtained from the available experimental data, intrinsically accounts for the onion skin theory (De Luca, Carlo J. \& Hostage, 2010) (Figure 8). It better replicates the MN membrane dynamics of the MN pool than Fuglevand-type phenomenological models (Fuglevand et al., 1993; Carriou et al., 2019), where the $\mathrm{MN}$-specific firing rates are predicted as a linear function of the synaptic input $I(t)$. Moreover, LIF models credibly replicate most of the MN membrane dynamics (Teeter et al., 2018) and allow accurate predictions of the MN pool behaviour (Figure 12, Figure 13), while they provide a simpler modelling approach and a more convenient framework for $\mathrm{MN}$ electrophysiological parameter assignment than comprehensive Hodgkin-Huxley-type MN models (Huxley \& Niedergerke, 1954; Huxley, 1957; Cisi \& Kohn, 2008; Negro \& Farina, 2011). As it relies on a physiologically realistic description of the MN pool architecture, the four-step approach is robust to systematic differences in the input experimental datasets. For example, it accurately predicted the individual $\mathrm{MN}$ firing behaviour of the $N_{r}$ MNs for all four datasets (Figure 12) despite the latter being obtained at different levels of muscle contractions, on different subjects and muscles and in different experimental approaches. The approach accurately predicted the $e N D_{N}$ of both $D T A_{35}$ and $H T A_{35}$ datasets from $N_{r}=32$ and 21 identified MNs respectively (Table 3), suggesting that the method is not sensitive to the number $N_{r}$ of identified MNs, providing that the $N_{r}$ identified MNs and their properties are homogeneously spread in the $\mathrm{MN}$ pool, as in datasets $D T A_{35}$ and $H T A_{35}$ where at least one $\mathrm{MN}$ is identified in the each 10\%-range of the rheobase domain. The accurate prediction of $e N D_{N}$ for the dataset $H G M_{30}$ in the time range $[4.7 ; 7.2] s$ (Figure 13 bottom-right plot) also suggests that the quality of the predictions is not sensitive to the hindlimb muscle investigated, providing that the $F^{t h}$ distribution is representative of the investigated muscle. 
bioRxiv preprint doi: https://doi.org/10.1101/2022.02.21.481337; this version posted February 22, 2022. The copyright holder for this preprint (which was not certified by peer review) is the author/funder, who has granted bioRxiv a license to display the preprint in perpetuity. It is made available under aCC-BY 4.0 International license.

903

904

905

906

907

908

909

910

911

912

913

914

915

916

917

918

919

920

921

922

923

924

\section{Limitations of the approach and potential improvements}

The two validations of the approach (Figure 12 and Figure 13) present some limitations. The local validation in Figure 12 only ensures that the method accurately estimates the $\mathrm{MN}$ firing behaviour for the fractions of the MN pool that were experimentally identified. This local validation alone does not inform on the accuracy of the predictions for the non-identified regions of the MN pool and must be coupled with a global validation of the MN pool behaviour by validating the predicted $e N D_{N}$. While the local validation was successful for the $H T A_{50}$ dataset (Figure 12), where less than $30 \%$ of the active $\mathrm{MN}$ pool is represented, it is inferred in Figure $13 \mathrm{C}$ that the individual MN firing rates were overestimated for the first half of the non-recorded $\mathrm{MN}$ pool. This local validation would be selfsufficient for experimental samples that contain a large and homogeneously spread population of identified MNs, obtained from decomposed fine-wire intramuscular electrode and HDEMG grid signals. The validation of the $e N D_{N}$ is performed in this study against an experimental force recorded by a transducer (green traces in Figure 13), which accounts for the force-generating activity of both the muscle of interest and the synergistic and antagonistic muscles crossing the ankle. The experimental force trace measured in the TA datasets may be an acceptable validation metric as the TA muscle is expected to explain most of the recorded ankle torque in dorsiflexion. The TA muscle is indeed the main dorsiflexor muscle with a muscle volume and maximum isometric force larger than the flexor hallucis longus muscle, which moment arm is besides not aligned with the dorsiflexion direction and mainly acts for ankle inversion. However, the gastrocnemius lateralis, soleus, and peroneus muscles acts synergistically with the GM muscle for ankle plantarflexion and the experimental torque recorded in dataset $H G M_{30}$ may not be representative of the $\mathrm{GM}$ muscle force-generation activity and may not be a suitable validation metric for $e N D_{N}$. In Figure 13, the decreasing $e N D_{N}$ and $e N D_{N_{r}}$ (orange and blue dotted traces) may accurately describe a gradually increasing sharing of the ankle torque between synergistic muscles initially taken by the GM muscle, which the constant validation trace does not capture. To answer such limitations, the predicted $e N D_{N}$ should be validated against a direct measure of muscle force, which can be performed as in other recent studies (Dick et al., 2017; Martinez-Valdes et al., 2021; Waasdorp et al., 2021) with ultrasound measurements of the muscle fascicle or of the muscle tendon concurrently obtained with (HD)EMG recordings of the muscle activity.

While the method predicts a list of simulated spike trains $s p_{j}^{s i m}(t)$ and a $e N D_{N}$ that more accurately describes the MN pool behaviour than the experimental $s p_{i}^{\exp }(t)$ and $e N D_{N_{r}}$, as discussed previously, the accuracy of these predictions (Figure 12 and Figure 13) remains sensitive to the distribution in the entire MN pool of the $N_{r}$ MNs identified experimentally, reported in the third column of Table 2. Because of the definition of the synaptic current input $I(t)$, the $e N D_{N}$ onset is defined by the 
recruitment time $t f_{N_{1}}^{1}$ of the lowest-threshold $\mathrm{MN} N_{1}$ identified experimentally, as shown in Figure 13, while the unknown firing behaviour for $t<f t_{N_{1}}^{1}$ of the non-identified MNs of rheobase lower than $I_{N_{1}}^{t h}$ is not captured. This is not an issue in samples of homogeneously distributed MNs like dataset $D T A_{35}$, where the $16^{\text {th }}$ smallest MN (first $4 \%$ of the pool of threshold-increasing MNs) is identified (Figure 5BC), Table 2) and the $e N D$ obtained from the 15 first MNs is not predicted during the short time range $[2.1,2.3] s$, where the whole muscle builds only 1\%MVC (top-left plot in Figure 13). However in heterogeneous or incomplete samples like dataset $H T A_{50}$ the lowest-threshold (232 ${ }^{\text {nd }}$, Table 2) identified $\mathrm{MN}$ identified is recruited at $t=t f_{N_{1}}^{1}=6.1 \mathrm{~s}$ and the approach wrongly returns a zero muscle neural drive $e N D=0$ for the time period $[1.6 ; 6.1] s$ where the muscle actually builds $20 \% \mathrm{MVC}$ during the ramp of contraction (bottom-left plot in Figure 13). To tackle this issue, the normalized force trace, which is non-zero for $t<f t_{N_{1}}^{1}$ and representative of the CSI in this time region, could be scaled to consistent values of synaptic current and used in lieu of the current definition of $I(t)$. Such synaptic input would also be more physiologically representative of the true synaptic current than $I(t)$, which $\operatorname{coher}_{N_{r}}$ value is below 1. However, this approach, which may not be suitable for non-isometric conditions, is not applicable in forward predictions of unknown whole muscle force from neural inputs. In pre-defined protocols of muscle contractions like trapezoidal ramps (Figure 2), the region $I(t<$ $f t_{N_{1}}^{1}$ ) could also be extrapolated by regression. Experimental samples of homogeneously distributed MNs are also required to derive realistic $S(j)$ and $I P(j)$ distributions with the four-step approach. As observed in Figure 12, ignoring in the derivation of $I(t), I P(j)$ and $S(j)$ the spike trains $s p_{i}^{\exp }(t)$ of the MNs that are representative of a large subset of the MN pool affects the accuracy of the predicted recruitment and firing behaviour of the MNs falling in that subset (Figure 12). More specifically, the non-physiological distribution $I P(j)[s]=5.6 \cdot 10^{-4} \cdot j^{0.80}$ was fitted to the experimental data of the 14 high-threshold MNs identified in dataset $H T A_{50}$, where the neural information of the $60 \%$ smallest MNs of the MN pool (Table 2 ) is lacking. Such $I P(j)$ distribution overestimates the LIF-predicted maximum firing frequency of low-threshold MNs, which explains the overestimation of the $e N D_{N}$ in Figure 13 (bottom-left plot, orange curve). Non-physiological predictions can be avoided by adding artificial data points consistent with other experiments or with the literature in the rheobase regions of the MN pool where no MNs were experimentally identified. For example, using an $I P(j)$ relationship consistent with a dataset of homogeneously distributed $\mathrm{MNs}\left(D T A_{35}\right)$ constrained the predicted maximum firing rates to physiological values and returned more accurate predictions of the $e N D_{N}$ (bottom-left plot, purple curve).

The four-step approach presents the following limitations and simplifications which make it currently unfeasible to mimic and predict certain neural mechanisms. First, it is inferred from the calibrated FIDFs in the $\left[t_{t r_{1}} ; t_{t r_{2}}\right]$ regions (Figure $9 \mathrm{~A}$ ) that the $\mathrm{MN}$ firing behaviour could be better predicted if the 
LIF IDF - I gain was lower for all MNs for large input currents. In LIF models, the IDF - I gain is mainly determined by the $\mathrm{MN}$ membrane capacitance $C=C_{m} \cdot S$, tuned by parameter calibration and sensitivity analysis in this study. To achieve a lower gain, a nonlinear $1^{\text {st }}$-order LIF model, a $2^{\text {nd }}$-order LIF model, a time-history-dependent $C$ parameter or a 3-parameter LIF model are options. However, considering the overall accurate predictions of the MN firing behaviours (Figure 12), its low computational cost and its modelling simplicity, the LIF model defined in this study was considered an adequate trade-off between accuracy and complexity and no other modelling approaches were pursued. A second limitation of the study is that, as discussed previously, the LIF parameters are tuned with typical cat data (Caillet et al. 2021) while the experimental MN spike trains were obtained from humans. While the normalized mathematical relationships relating the MN electrophysiological parameters of the LIF model $\left(R, C, \tau, \Delta V_{t h}\right)$ can be extrapolated between mammals (Caillet et al. 2021) and thus to humans, no experimental data is yet available to scale these relationships to typical human data. A further limitation is that, in its current form, the method disregards the synaptic noise $S N(t)$ specific to each MN and the inter-spike variability (ISV). The synaptic current $I(t)$ is simplified as the common synaptic drive to the $\mathrm{MN}$ pool. Considering that the $\mathrm{MN}$ pool and the $\mathrm{MU}$ neuromechanical mechanisms are expected to filter the $\mathrm{MN}$-specific $S N(t)$, this simplification is adequate for accurate predictions of the $e N D_{N}$ and of the whole muscle force (Farina, 2015). Sometimes modelled as a random Gaussian-like event (Fuglevand 1993), the ISV can be obtained as the response to a $S N(t)$ white noise added to the current definition of the synaptic current as $I(t)=\operatorname{CSI}(t)+\operatorname{SN}(t)$. The relative proportion of $C S I$ and $S N$ in \% of the variance of the total synaptic current can be approximated from Figure 2A in Negro et al. (2016). Accounting for $S N(t)$ might improve the accuracy of the predicted firing behaviour of the largest MNs, for which the largest $\Delta f t^{1}$ and $n R M S E$ and lowest $r^{2}$ values were obtained in Figure 12 for all four datasets. These MNs are recruited at a rheobase close to the nearly constant common synaptic current $I_{\text {plat }}$ maintained during the plateau of force in the time range $\left[t_{t r_{2}} ; t_{t r_{3}}\right]\left(I_{t h} \approx I_{\text {plat }}\right)$ and their firing behaviour and recruitment dynamics are dominantly dictated by random fluctuations of $S N(t)$. The capacity of the synaptic drive to generate larger synaptic currents $I(t)$ in larger MNs (Heckman 2012) could be accounted for with a MN size-specific scaling factor assigned to the $I(t)$ trace obtained in Figure 7 (B). The MN-specific decrease in current threshold between recruitment and derecruitment phases shows a $\mathrm{MN}$-specific random behaviour around a global linear tendency across the complete MN pool (Figure 4B), which could be captured by adding a randomized component to the value of the $\mathrm{MN}$ input resistance which is increased between recruitment and derecruitment phases (see Methods). Such an approach would better predict the derecruitment times of individual MNs and the derecruitment time period of the predicted $e N D_{N}$. 
An additional limitation is that the typical $F^{t h}(j)$ and $I_{t h}(j)$ threshold distributions, derived for mapping the $N_{r}$ identified MNs to the complete $\mathrm{MN}$ pool (Figure 5) and for scaling the CSI to physiological values of $I(t)$ (Figure 7), were obtained from studies which relied on experimental samples of MN populations of small size. These source studies therefore did not ensure that the largest and lowest values were identified and reported, and that the identification process was not biased towards a specific subset of the MN pool, such as larger MNs. In such cases, the true threshold distributions would be more skewed and spread over a larger range of values, as discussed in Caillet et al. (2021), than the distributions reported in Figure 5 and Figure 7. The $F^{\text {th }}(j)$ distribution is besides muscle-specific (Heckman 2012) with large hindlimb muscles being for example recruited over a larger range of MVC than hand muscles. However, enough data is reported in the literature to build the $F^{t h}(j)$ distributions for the TA and first dorsal interossei human muscles only. MN rheobase cannot be recorded in humans and the $I_{t h}(j)$ distribution in Figure 7A was obtained from cat experimental data obtained from various hindlimb muscles. For these limitations, the two first steps of this approach could be made subject- species- and muscle-specific by calibrating the $F^{\text {th }}(j)$ and $I^{\text {th }}(j)$ described as the 3-parameter power functions defined in this study.

\section{Relevance for neuromuscular modelling}

In this study, a MN-driven muscle model of in-parallel Hill-type actuators describing the MUs (Figure 3 ) is used to perform forward predictions of the whole muscle force in voluntary isometric contraction. Each Hill-type actuator predicts the excitation, activation and contraction dynamics of each MU constituting the muscle, i.e. the MU neural, action potential, calcium transient, activation and contraction behaviour. The whole muscle force is computed as the linear summation of the predicted MU forces. The MN-driven muscle model either takes as inputs the $N_{r}$ individual MN spike trains $s p_{i}^{e x p}(t)$ identified experimentally (blue trace in Figure 16) or the $N$ simulated spike trains $s p_{j}^{s i m}(t)$ (red trace in Figure 16).

This approach advances the state-of-the-art in neuromusculoskeletal modelling on several aspects. The MN-driven muscle model (Figure 3 ) is driven by an input vector of experimental MN spike trains, so extending the classic single input EMG-driven approach, in which the individual MN contributions are lumped into a unique neural control to the muscle, taken as the rectified-normalized-filtered envelope of recorded bipolar EMGs (bEMGs) (Hof \& Van den Berg, 1981; Zajac Felix, 1989; Lloyd \& Besier, 2003). bEMGs are also challenging to interpret (De Luca, Carlo J., 1997) and come with intrinsic experimental limitations that are circumvented by HDEMG and/or intramuscular approaches (Staudenmann et al., 2006; Mesin et al., 2009; Varrecchia et al., 2021), for which current 
bioRxiv preprint doi: https://doi.org/10.1101/2022.02.21.481337; this version posted February 22, 2022. The copyright holder for this preprint (which was not certified by peer review) is the author/funder, who has granted bioRxiv a license to display the preprint in perpetuity. It is made available under aCC-BY 4.0 International license.

decomposition techniques are a reliable process across operators (Hug, Avrillon et al., 2021). The MNdriven muscle model (Figure 3) also represents an improvement with respect to recent studies which merged the individual $\mathrm{MN}$ spike trains obtained from decomposed HDEMG or intramuscular recordings into a unique neural control, the $C S T_{N_{r}}$ given as input to Hill-type (Sartori et al., 2017; Kapelner et al., 2020) and twitch-type (Thompson et al., 2018) muscle models. As in bEMG-driven approaches, the $\operatorname{CST}_{N_{r}}$ loses the $\mathrm{MN}$-specific information of $\mathrm{MN}$ neural behaviour, recruitment dynamics and contributions to the neural drive. The multi-MU model in Figure 3 expands the classic multiscale approach of modelling a whole muscle as a single equivalent sarcomere or MU (Zajac Felix, 1989). Such multiscale models usually (1) cannot explicitly model the dynamics of MU recruitment, (2) disregard the continuous distribution of $\mathrm{MU}$ properties in the $\mathrm{MU}$ pool that typically affects the $\mathrm{MN}$ firing behaviour (Caillet et al., 2021), the calcium dynamics in the sarcolemma (Baylor \& Hollingworth, 2003), the cross-bridge cycling dynamics (Rockenfeller \& Günther, 2018), the force-velocity relationship (Alcazar et al., 2019) and the MU maximum isometric force distribution, and (3) cannot account for the passive properties of the intramuscular matrix of passive tissues (Herzog, 2019). Despite relevant modelling work in multi-scale modelling (Hatze, 1977; Hatze, 1980; Dorgan \& O'Malley, 1997; Song et al., 2008; Dick et al., 2017; Hussein et al., 2021), multi-MU muscle models like the one proposed in Figure 3 provide a more convenient framework to answer the above limitations. The multi-MU models available in the literature (Hatze, 1980; Legreneur et al., 1996; Callahan et al., 2013; Hamouda et al., 2016; Potvin \& Fuglevand, 2017; Raikova et al., 2018; Carriou et al., 2019) were however never assessed with experimental neural inputs or validated against synchronously recorded experimental data, and simply recreated the recruitment and firing $\mathrm{MN}$-specific behaviour of the modelled individual MUs, which are intrinsic to the experimental sample of $N_{r}$ spike trains obtained from signal decomposition. The MN-driven multi-MU muscle model in Figure 3 therefore diverges from past approaches by predicting and experimentally validating the whole muscle force using a physiologically realistic model of a $\mathrm{MU}$ population receiving an experimental $\mathrm{MN}$ spike trains as a vector of inputs.

The $N$-MU model, that receives as inputs the $N$ spike trains $s p_{j}^{s i m}(t)$ generated by the four-step approach, predicted the whole muscle force more accurately (Table 3) than the $N_{r}$-MU model receiving the $N_{r}$ individual $\mathrm{MN}$ spike trains $s p_{i}^{e x p}(t)$ (Figure 16). As discussed previously, the $N_{r}$ identified MNs are not representative of the distribution of $\mathrm{MN}$ and $\mathrm{MU}$ properties in $\mathrm{MU}$ pool (Figure 5) and of the neural activity of the complete MN pool (Figure 13). In neuromuscular modelling, this affects the realistic assignment of MU properties, such as MU type and MU maximum isometric force, to the sample of $N_{r}$ MUs and limits the interpretations of the $N_{r}$-MU muscle model predictions. The $N_{r}$-MU model besides underestimates the whole muscle in the regions of low muscle activity where 
dominantly low-threshold MUs are recruited but are under-represented in the experimental sample. A similar limitation arises for the studies using the $C S T_{N_{r}}$ as input to their muscle models. Describing the complete population of MUs and accounting for the whole distribution of $\mathrm{MU}$ neuromechanical properties in the muscle with the $N$-MU model tackles the limitations related to the $N_{r}-\mathrm{MU}$ model. A few other studies have predicted whole muscle forces with a collection of $N$ MU Hill-type (Legreneur et al., 1996; Callahan et al., 2013) Hill-Huxley-type (Carriou et al., 2019) or twitch-type (Raikova et al., 2018) muscle models from the neuromuscular activity of complete pools of MUs, the MN firing behaviour and recruitment dynamics of which were modelled with the Fuglevand's formalism. Contrary to these studies which considered an artificial synaptic input and performed indirect validations of the predicted forces against results from other models, the whole muscle force predictions in Figure 13 and Figure 16 were obtained from and validated against experimental data. The $N$-MU model besides receives the $N$ validated neural inputs from the four-step approach that is more realistic and advanced than the Fuglevand's formalism, as discussed previously.

The $\mathrm{N}$-MU neuromuscular model defined in this study relies on realistic and mechanistic descriptions of the MU-specific neural and mechanical mechanisms and on physiological parameters that can be interpreted and measured experimentally in mammals. The isometric muscle force trace can therefore be accurately predicted in this approach without relying on any parameter calibration except the MN size in step 3. The neuromuscular model proposed in this study is thus more suitable for investigating the muscle neuromechanics and confidently reconciling spinal motor control and muscle contraction than typical EMG-driven models, the neural parameters of which do not have a direct physiological correspondence and must be calibrated to match experimental joint torques (Lloyd \& Besier, 2003; Sartori et al., 2012; Pizzolato et al., 2015). 


\section{REFERENCES}

1094

1095

Alcazar, J.,Csapo, R.,Ara, I.,Alegre, L.M.(2019) On the shape of the force-velocity relationship in skeletal muscles: The linear, the hyperbolic, and the double-hyperbolic. Front. Physiol., 10.

Andreassen S, Arendt-Nielsen L (1987) Muscle fibre conduction velocity in motor units of the human anterior tibial muscle: A new size principle parameter. J. Physiol. 391:561-571.

Ankit, R. (2020) WebPlotDigitizer. 4.4 https://automeris.io/WebPlotDigitizer.

Bakels R, Kernell D (1993) Matching between motoneurone and muscle unit properties in rat medial gastrocnemius. J. Physiol. 463:307-324.

Ballantyne BT, Kukulka CG, Soderberg GL (1993) Motor unit recruitment in human medial gastrocnemius muscle during combined knee flexion and plantarflexion isometric contractions. Experimental brain research 93:492-498.

Baylor SM, Hollingworth S (2003) Sarcoplasmic reticulum calcium release compared in slow-twitch and fast-twitch fibres of mouse muscle. J.Physiol.(Lond.) 551:125-138.

Boe SG, Dalton BH, Harwood B, Doherty TJ, Rice CL (2009) Inter-rater reliability of motor unit number estimates and quantitative motor unit analysis in the tibialis anterior muscle. Clinical neurophysiology 120:947-952.

Bondarenko VE, Szigeti GP, Bett GC, Kim S, Rasmusson RL (2004) Computer model of action potential of mouse ventricular myocytes. American Journal of Physiology-Heart and Circulatory Physiology 287:H1378-H1403.

Caillet A, Phillips A, Farina D, Modenese L (2021) Mathematical relationships between spinal motoneuron properties. bioRxiv .

Callahan DM, Umberger BR, Kent-Braun JA (2013) A computational model of torque generation: Neural, contractile, metabolic and musculoskeletal components. PloS one 8:e56013.

Carriou V, Boudaoud S, Laforet J, Mendes A, Canon F, Guiraud D (2019) Multiscale hill-type modeling of the mechanical muscle behavior driven by the neural drive in isometric conditions. Computers in biology and medicine 115:103480.

Chen C, Yu Y, Ma S, Sheng X, Lin C, Farina D, Zhu X (2020) Hand gesture recognition based on motor unit spike trains decoded from high-density electromyography. Biomedical signal processing and control 55:101637.

Christensen E (1959) American journal of physical medicine \& rehabilitation. American Journal of Physical Medicine \& Rehabilitation 38:65-78.

Cisi RRL, Kohn AF (2008) Simulation system of spinal cord motor nuclei and associated nerves and muscles, in a web-based architecture. J Comput Neurosci 25:520-542.

Connelly DM, Rice CL, Roos MR, Vandervoort AA (1999) Motor unit firing rates and contractile properties in tibialis anterior of young and old men. J.Appl.Physiol. 87:843-852. 
De Luca CJ, Lefever RS, Mccue MP, Xenakis AP (1982) Behaviour of human motor units in different muscles during linearly varying contractions. J. Physiol 329:113-128.

De Luca CJ, Hostage EC (2010) Relationship between firing rate and recruitment threshold of motoneurons in voluntary isometric contractions. J.Neurophysiol. 104:1034-1046.

De Luca CJ (1997) The use of surface electromyography in biomechanics. Journal of applied biomechanics 13:135-163.

Del Vecchio A, Holobar A, Falla D, Felici F, Enoka RM, Farina D (2020) Tutorial: Analysis of motor unit discharge characteristics from high-density surface EMG signals. Journal of Electromyography and Kinesiology 53:102426.

Del Vecchio A, Casolo A, Negro F, Scorcelletti M, Bazzucchi I, Enoka R, Felici F, Farina D (2019) The increase in muscle force after 4 weeks of strength training is mediated by adaptations in motor unit recruitment and rate coding. J Physiol 597:1873-1887.

Dengler R, Stein RB, Thomas CK (1988) Axonal conduction velocity and force of single human motor units. Muscle \& Nerve: Official Journal of the American Association of Electrodiagnostic Medicine 11:136-145.

Desmedt JE, Godaux E (1977) Ballistic contractions in man: Characteristic recruitment pattern of single motor units of the tibialis anterior muscle. The Journal of physiology 264:673-693.

Dick TJM, Biewener AA, Wakeling JM (2017) Comparison of human gastrocnemius forces predicted by hill-type muscle models and estimated from ultrasound images. Journal of experimental biology 220:1643-1653.

Dideriksen JL, Enoka RM, Farina D (2011) Neuromuscular adjustments that constrain submaximal EMG amplitude at task failure of sustained isometric contractions. Journal of Applied Physiology 111:485-494.

Dideriksen JL, Farina D, Baekgaard M, Enoka RM (2010) An integrative model of motor unit activity during sustained submaximal contractions. Journal of Applied Physiology 108:1550-1562.

Dong Y, Mihalas S, Russell A, Etienne-Cummings R, Niebur E (2011) Estimating parameters of generalized integrate-and-fire neurons from the maximum likelihood of spike trains. Neural Comput. 23:2833-2867.

Dorgan SJ, O'Malley MJ (1997) A nonlinear mathematical model of electrically stimulated skeletal muscle. T-RE 5:179-194.

Fang C, He B, Wang Y, Cao J, Gao S (2020) EMG-centered multisensory based technologies for pattern recognition in rehabilitation: State of the art and challenges. Biosensors 10:85.

Farina D, Vujaklija I, Brånemark R, Bull AMJ, Dietl H, Graimann B, Hargrove LJ, Hoffmann K, Huang H, Ingvarsson T, Janusson HB, Kristjánsson K, Kuiken T, Micera S, Stieglitz T, Sturma A, Tyler D, Weir RFf, Aszmann OC (2021) Toward higher-performance bionic limbs for wider clinical use. Nature biomedical engineering 1-13. 
Farina D, Vujaklija I, Sartori M, Kapelner T, Negro F, Jiang N, Bergmeister K, Andalib A, Principe J, Aszmann OC (2017) Man/machine interface based on the discharge timings of spinal motor neurons after targeted muscle reinnervation. Nature biomedical engineering 1:1-12.

Farina D, Negro F (2015) Common synaptic input to motor neurons, motor unit synchronization, and force control. Exerc.Sport Sci.Rev. 43:23-33.

Farina D, Negro F, Dideriksen JL (2014) The effective neural drive to muscles is the common synaptic input to motor neurons. The Journal of physiology 592:3427-3441.

Feiereisen P, Duchateau J, Hainaut K (1997) Motor unit recruitment order during voluntary and electrically induced contractions in the tibialis anterior. Experimental brain research 117.123.

Feinstein B, Lindegård B, Nyman E, Wohlfart G (1955) Morphologic studies of motor units in normal human muscles. Cells Tissues Organs (Print) 23:127-142.

Felici F, Del Vecchio A (2020) Surface electromyography: What limits its use in exercise and sport physiology? Frontiers in neurology 11:578504.

Fleshman JW, Munson JB, Sypert GW, Friedman WA (1981) Rheobase, input resistance, and motorunit type in medial gastrocnemius motoneurons in the cat. J Neurophysiol 46:1326-1338.

Foehring RC, Sypert GW, Munson JB (1987) Motor-unit properties following cross-reinnervation of cat lateral gastrocnemius and soleus muscles with medial gastrocnemius nerve. II. influence of muscle on motoneurons. J Neurophysiol 57:1227-1245.

Foehring RC, Sypert GW, Munson JB (1986) Properties of self-reinnervated motor units of medial gastrocnemius of cat. II. axotomized motoneurons and time course of recovery. J Neurophysiol 55:947-965.

Fohlmeister JF (2009) A nerve model of greatly increased energy-efficiency and encoding flexibility over the Hodgkin-Huxley model. Brain Res. 1296:225-233.

Freund HJ, Budingen HJ, Dietz V (1975) Activity of single motor units from human forearm muscles during voluntary isometric contractions. J.Neurophysiol. 38:933-946.

Fuglevand AJ, Winter DA, Patla AE (1993) Models of recruitment and rate coding organization in motor-unit pools. Journal of neurophysiology 70:2470-2488.

Gardiner PF (1993) Physiological properties of motoneurons innervating different muscle unit types in rat gastrocnemius. J Neurophysiol 69:1160-1170.

Garnett RAF, O'Donovan MJ, Stephens JA, Taylor A (1979) Motor unit organization of human medial gastrocnemius. J. Physiol. 287:33-43.

Gentet L, Stuart GJ, Clements JD (2000) Direct measurement of specific membrane capacitance in neurons. Biophys. J. 79:314-320.

Gooch CL, Doherty TJ, Chan KM, Bromberg MB, Lewis RA, Stashuk DW, Berger MJ, Andary MT, Daube JR (2014) Motor unit number estimation: A technology and literature review. Muscle Nerve 50:884893. 
Gustafsson, Pinter MJ (1984) An investigation of threshold properties among cat spinal alphamotoneurones. J. Physiol. 357:453-483.

Hamouda A, Kenney L, Howard D (2016) Dealing with time-varying recruitment and length in hill-type muscle models. Journal of biomechanics 49:3375-3380.

Handsfield GG, Meyer CH, Hart JM, Abel MF, Blemker SS (2014) Relationships of 35 lower limb muscles to height and body mass quantified using MRI. J.Biomech. 47:631-638.

Hatze H (1980) Myocybernetic Control Models of Skeletal Muscle - Characteristics and Applications. University of South Africa Press, Pretoria, .

Hatze H (1977) A myocybernetic control model of skeletal muscle. Biological cybernetics 25:103-119.

Heckman CJ, Enoka RM (2012) Motor unit. Compr. Physiol. 2:2629-2682.

Henneman E (1985) The size-principle: A deterministic output emerges from a set of probabilistic connections. J. Exp. Biol. 115:105-112.

Henneman E (1981) Recruitment of motoneurons : The size principle. Motor Unit Types, Recruitment and Plasticity in Health and Disease 26-60.

Henneman E, Clamann HP, Gillies JD, Skinner RD (1974) Rank order of motoneurons within a pool: Law of combination. J Neurophysiol 37:1338-1349.

Henneman E, Somjen G, Carpenter DO (1965a) Excitability and inhibitibility of motoneurons of different sizes. J Neurophysiol 28:599-620.

Henneman E, Somjen G, Carpenter DO (1965b) Functional significance of cell size in spinal motoneurons. J Neurophysiol 28:560-580.

Henneman E (1957) Relation between size of neurons and their susceptibility to discharge. Science 126:1345-1347.

Henriksson-Larsén K (1985) Distribution, number and size of different types of fibres in whole crosssections of female $m$ tibialis anterior. an enzyme histochemical study. Acta Physiol.Scand. 123:229235.

Henriksson-Larsén KB, Lexell J, Sjöström M (1983) Distribution of different fibre types in human skeletal muscles. I. method for the preparation and analysis of cross-sections of whole tibialis anterior. Histochem.J. 15:167-178.

Héroux ME, Dakin CJ, Luu BL, Inglis JT, Blouin J (2014) Absence of lateral gastrocnemius activity and differential motor unit behavior in soleus and medial gastrocnemius during standing balance. J.Appl.Physiol. 116:140-148.

Herzog W (2019) The problem with skeletal muscle series elasticity. BMC biomedical engineering $1: 28$.

Highlander, M. M.,Allen, J.M.,Elbasiouny, S.M.(2020) Meta-analysis of biological variables' impact on spinal motoneuron electrophysiology data. Journal of Neurophysiology, 123, 1380. 
Hof AL, Van den Berg J (1981) EMG to force processing I: An electrical analogue of the hill muscle model. Journal of biomechanics 14:747,755-753,758.

Holobar A, Farina D (2014) Blind source identification from the multichannel surface electromyogram. PM 35:R143-R165.

Hourigan ML, McKinnon NB, Johnson M, Rice CL, Stashuk DW, Doherty TJ (2015) Increased motor unit potential shape variability across consecutive motor unit discharges in the tibialis anterior and vastus medialis muscles of healthy older subjects. Clinical Neurophysiology 126:2381-2389.

Hug F, Avrillon S, Del Vecchio A, Casolo A, Ibanez J, Nuccio S, Rossato J, Holobar A, Farina D (2021) Analysis of motor unit spike trains estimated from high-density surface electromyography is highly reliable across operators. Journal of Electromyography and Kinesiology 58:102548.

Hug F, Del Vecchio A, Avrillon S, Farina D, Tucker K (2021) Muscles from the same muscle group do not necessarily share common drive: Evidence from the human triceps surae. J.Appl.Physiol. 130:342354.

Hussein M, Shebl S, Elnemr R, Elkaranshawy H (2021) A new muscle activation dynamics model, that simulates the calcium kinetics and incorporates the role of store-operated calcium entry channels, to enhance the electromyography-driven hill-type models. J.Biomech.Eng. 144:011002.

Huxley AF (1957) Muscle structure and theories of contraction. Prog.Biophys.Biophys.Chem 7:255318.

Huxley AF, Niedergerke R (1954) Structural changes in muscle during contraction: Interference microscopy of living muscle fibres. Nature 173:971-973.

Izhikevich EM (2004) Which model to use for cortical spiking neurons? IEEE Trans.Neural Networks 15:1063-1070.

Jordanić M, Rojas-Martínez M, Mañanas MA, Alonso JF (2016) Prediction of isometric motor tasks and effort levels based on high-density EMG in patients with incomplete spinal cord injury. J. Neural Eng. 13:046002.

Kallio J, Søgaard K, Avela J, Komi PV, Selänne H, Linnamo V (2013) Motor unit firing behaviour of soleus muscle in isometric and dynamic contractions. PloS one 8:e53425.

Kapelner T, Sartori M, Negro F, Farina D (2020) Neuro-musculoskeletal mapping for man-machine interfacing. Scientific reports 10:1-10.

Kernell D, Zwaagstra B (1989) Dendrites of cat's spinal motoneurones: Relationship between stem diameter and predicted input conductance. J. Physiol. 413:255-269.

Kernell D, Monster AW (1981) Threshold current for repetitive impulse firing in motoneurones innervating muscle fibres of different fatigue sensitivity in the cat. Brain Res. 229:193-196.

Kernell D (1966) Input resistance, electrical excitability, and size of ventral horn cells in cat spinal cord. Science 152:1637-1640. 
Kim H, Kim M (2018) PyMUS: Python-based simulation software for virtual experiments on motor unit system. Frontiers in neuroinformatics 12:15.

Kisiel-Sajewicz K, Marusiak J, Rojas-Martínez M, Janecki D, Chomiak S, Kamiński Ł, Mencel J, Mañanas MÁ, Jaskólski A, Jaskólska A (2020) High-density surface electromyography maps after computeraided training in individual with congenital transverse deficiency: A case study. BMC musculoskeletal disorders 21:682.

Krawitz S, Fedirchuk B, Dai Y, Jordan LM, McCrea DA (2001) State-dependent hyperpolarization of voltage threshold enhances motoneurone excitability during fictive locomotion in the cat. The Journal of physiology 532:271-281.

Legreneur P, Morlon B, Van Hoecke J (1996) Simulation of in situ soleus isometric force output as a function of neural excitation. Journal of biomechanics 29:1455-1462.

Lloyd DG, Besier TF (2003) An EMG-driven musculoskeletal model to estimate muscle forces and knee joint moments in vivo. Journal of Biomechanics 36:765-776.

Maeda N, Komiya M, Nishikawa Y, Morikawa M, Tsutsumi S, Tashiro T, Fukui K, Kimura H, Urabe Y (2021) Effect of acute static stretching on the activation patterns using high-density surface electromyography of the gastrocnemius muscle during ramp-up task. Sensors (Basel, Switzerland) 21:4841.

Manuel M, Chardon M, Tysseling V, Heckman CJ (2019) Scaling of motor output, from mouse to humans. Physiology (Bethesda, Md.) 34:5-13.

Martinez-Valdes, E.,Negro, F.,Botter, A.,Pincheira, P.A.,Cerone, G.L.,Falla, D.,Lichtwark, G.A.,Cresswell, A.G.(2021) Modulations in motor unit discharge are related to changes in fascicle length during isometric contractions.

McNeil CJ, Doherty TJ, Stashuk DW, Rice CL (2005) Motor unit number estimates in the tibialis anterior muscle of young, old, and very old men. Muscle \& Nerve: Official Journal of the American Association of Electrodiagnostic Medicine 31:461-467.

Mesin L, Merletti R, Rainoldi A (2009) Surface EMG: The issue of electrode location. Journal of Electromyography and Kinesiology 19:719-726.

Munson JB, Foehring RC, Lofton SA, Zengel JE, Sypert GW (1986) Plasticity of medial gastrocnemius motor units following cordotomy in the cat. Journal of neurophysiology 55:1454.

Negro F, Muceli S, Castronovo AM, Holobar A, Farina D (2016) Multi-channel intramuscular and surface EMG decomposition by convolutive blind source separation. JNE 13:026027.

Negro F, Yavuz U, Farina D (2016) The human motor neuron pools receive a dominant slow-varying common synaptic input. J. Physiol. 594:5491-5505.

Negro F, Farina D (2011) Decorrelation of cortical inputs and motoneuron output. Journal of Neurophysiology 106:2688-2697. 
Nishikawa Y, Watanabe K, Holobar A, Maeda N, Maruyama H, Tanaka S (2021) Identification of the laterality of motor unit behavior in female patients with parkinson's disease using high-density surface electromyography. Eur.J.Neurosci. 53:1938-1949.

Oliveira AS, Negro F (2021) Neural control of matched motor units during muscle shortening and lengthening at increasing velocities. Journal of applied physiology (1985) 130:1798-1813.

Piasecki M, Ireland A, Coulson J, Stashuk DW, Hamilton-Wright A, Swiecicka A, Rutter MK, McPhee JS, Jones DA (2016) Motor unit number estimates and neuromuscular transmission in the tibialis anterior of master athletes: Evidence that athletic older people are not spared from age-related motor unit remodeling. Physiological reports 4:e12987.

Pilkar R, Momeni K, Ramanujam A, Ravi M, Garbarini E, Forrest GF (2020) Use of surface EMG in clinical rehabilitation of individuals with $\mathrm{SCl}$ : Barriers and future considerations. Front. Neurol. 11:1680.

Pizzolato C, Lloyd DG, Sartori M, Ceseracciu E, Besier TF, Fregly BJ, Reggiani M (2015) CEINMS: A toolbox to investigate the influence of different neural control solutions on the prediction of muscle excitation and joint moments during dynamic motor tasks. J.Biomech. 48:3929-3936.

Potvin JR, Fuglevand AJ (2017) A motor unit-based model of muscle fatigue. PLoS computational biology 13:e1005581.

Power GA, Dalton BH, Behm DG, Vandervoort AA, Doherty TJ, Rice CL (2010) Motor unit number estimates in masters runners: Use it or lose it. Med.Sci.Sports Exerc. 42:1644-1650.

Raikova R, Celichowski J, Angelova S, Krutki P (2018) A model of the rat medial gastrocnemius muscle based on inputs to motoneurons and on an algorithm for prediction of the motor unit force. Journal of neurophysiology 120:1973-1987.

Rajagopal A, Dembia CL, DeMers MS, Delp DD, Hicks JL, Delp SL (2016) Full-body musculoskeletal model for muscle-driven simulation of human gait. TBME 63:2068-2079.

Rockenfeller R, Günther M (2018) Inter-filament spacing mediates calcium binding to troponin: A simple geometric-mechanistic model explains the shift of force-length maxima with muscle activation. Journal of theoretical biology 454:240-252.

Röhrle O, Yavuz U, Klotz T, Negro F, Heidlauf T (2019) Multiscale modeling of the neuromuscular system: Coupling neurophysiology and skeletal muscle mechanics. Wiley interdisciplinary reviews. Systems biology and medicine 11:e1457.

Sartori M, Yavuz U, Farina D (2017) In vivo neuromechanics: Decoding causal motor neuron behavior with resulting musculoskeletal function. Scientific reports 7:13465-14.

Sartori M, Reggiani M, Farina D, Lloyd DG (2012) EMG-driven forward-dynamic estimation of muscle force and joint moment about multiple degrees of freedom in the human lower extremity. PloS one 7:e52618.

Seth A, Hicks JL, Uchida TK, Habib A, Dembia CL, Dunne JJ, Ong CF, Demers MS, Rajagopal A, Millard M, Hamner SR, Arnold EM (2018) OpenSim: Simulating musculoskeletal dynamics and neuromuscular control to study human and animal movement. PLoS computational biology 14:e1006223. 
Smit JE, Hanekom T, Hanekom JJ (2008) Predicting action potential characteristics of human auditory nerve fibres through modification of the hodgkin-huxley equations. S.Afr.J.Sci. 104:284-292.

Song D, Raphael G, Lan N, Loeb GE (2008) Computationally efficient models of neuromuscular recruitment and mechanics. Journal of neural engineering 5:175-184.

Staudenmann D, Kingma I, Daffertshofer A, Stegeman DF, van Dieën JH (2006) Improving EMG-based muscle force estimation by using a high-density EMG grid and principal component analysis. IEEE Transactions on Biomedical Engineering 53:712-719.

Teeter C, Iyer R, Menon V, Gouwens N, Feng D, Berg J, Szafer A, Cain N, Zeng H, Hawrylycz M, Koch C, Mihalas $S$ (2018) Generalized leaky integrate-and-fire models classify multiple neuron types. Nat Commun 9:1-15.

Thompson CK, Negro F, Johnson MD, Holmes MR, McPherson LM, Powers RK, Farina D, Heckman CJ (2018) Robust and accurate decoding of motoneuron behaviour and prediction of the resulting force output. J.Physiol.(Lond.) 596:2643-2659.

Trojaborg W, Kaufmann P, Gooch CL (2002) Motor unit estimate number in the anterior tibial muscle: Normative data versus findings in critically ill patients in intensive care units. Journal of clinical neuromuscular disease 3:139-142.

Van Cutsem M, Feiereisen P, Duchateau J, Hainaut K (1997) Mechanical properties and behaviour of motor units in the tibialis anterior during voluntary contractions. Can. J. Appl. Physiol. 22:585-597.

Van Cutsem M, Duchateau J, Hainaut K (1998) Changes in single motor unit behaviour contribute to the increase in contraction speed after dynamic training in humans. J.Physiol.(Lond.) 513:295-305.

Vander Linden DW, Kukulka CG, Soderberg GL (1991) The effect of muscle length on motor unit discharge characteristics in human tibialis anterior muscle. Experimental brain research 84:210-218.

Varrecchia T, Ranavolo A, Conforto S, De Nunzio AM, Arvanitidis M, Draicchio F, Falla D (2021) Bipolar versus high-density surface electromyography for evaluating risk in fatiguing frequency-dependent lifting activities. Appl.Ergon. 95:103456.

Vieira TM, Loram ID, Muceli S, Merletti R, Farina D (2012) Recruitment of motor units in the medial gastrocnemius muscle during human quiet standing: Is recruitment intermittent? what triggers recruitment? J.Neurophysiol. 107:666-676.

Waasdorp R, Mugge W, Vos HJ, De Groot JH, Verweij MD, De Jong N, Schouten AC, Daeichin V (2021) Combining ultrafast ultrasound and high-density EMG to assess local electromechanical muscle dynamics: A feasibility study. Access 9:1.

Watanabe K, Vieira TM, Gallina A, Kouzaki M, Moritani T (2021) Novel insights into biarticular muscle actions gained from high-density electromyogram. Exercise and sport sciences reviews 49:179-187.

Watanabe RN, Magalhães FH, Elias LA, Chaud VM, Mello EM, Kohn AF (2013) Influences of premotoneuronal command statistics on the scaling of motor output variability during isometric plantar flexion. Journal of Neurophysiology 110:2592-2606. 
bioRxiv preprint doi: https://doi org/10.1101/2022 02.21.481337; this version posted February 22,2022 . The copyright holder for this preprint (which was not certified by peer review) is the author/funder, who has granted bioRxiv a license to display the preprint in perpetuity. It is made available under aCC-BY 4.0 International license.

1379 Xiong GX, Zhang JW, Hong Y, Guan Y, Guan H (2008) Motor unit number estimation of the tibialis 1380 anterior muscle in spinal cord injury. Spinal Cord 46:696-702.

1381 Zajac Felix E (1989) Muscle and tendon: Properties, models, scaling, and application to biomechanics and motor control. Critical reviews in biomedical engineering 17:359-411.

Zengel JE, Reid SA, Sypert GW, Munson JB (1985) Membrane electrical properties and prediction of motor-unit type of medial gastrocnemius motoneurons in the cat. J Neurophysiol 53:1323-1344.

Zot HG, Hasbun JE (2016) Modeling Ca2 -bound troponin in excitation contraction coupling. Frontiers in physiology 7:406. 